\title{
Structured outflow from a dynamo active accretion disc
}

\author{
B. von Rekowski ${ }^{\star}$, A. Brandenburg ${ }^{\star \star}$, W. Dobler ${ }^{\star \star}$, and A. Shukurov \\ Department of Mathematics, University of Newcastle, Newcastle upon Tyne NE1 7RU, UK \\ Received 25 May 2000 / Accepted 14 November 2002

\begin{abstract}
We present an axisymmetric numerical model of a dynamo active accretion disc. If the dynamo-generated magnetic field in the disc is sufficiently strong (close to equipartition with thermal energy), a fast magneto-centrifugally driven outflow develops within a conical shell near the rotation axis, together with a slower pressure driven outflow from the outer parts of the disc as well as around the axis. Our results show that a dynamo active accretion disc can contribute to driving an outflow even without any external magnetic field. The fast outflow in the conical shell is confined by the azimuthal field which is produced by the dynamo in the disc and advected to the disc corona. This part of the outflow has high angular momentum and is cooler and less dense than its surroundings. The conical shell's half-opening angle is typically about $30^{\circ}$ near the disc and decreases slightly with height. The slow outflow is hotter and denser.
\end{abstract}

Key words. ISM: jets and outflows - accretion, accretion disks - magnetic fields - MHD

\section{Introduction}

The importance of magnetic fields for disc accretion is widely recognized (e.g., Mestel 1999), and the turbulent dynamo is believed to be a major source of magnetic fields in accretion discs (Pudritz 1981a, 1981b; Stepinski \& Levy 1988; Brandenburg et al. 1995; Hawley et al. 1996). A magnetic field anchored in the disc is further considered to be a major factor in launching and collimating a wind in young stellar objects and active galactic nuclei (Blandford \& Payne 1982; Pelletier \& Pudritz 1992); see Königl \& Pudritz (2000) for a recent review of stellar outflows. Yet, most models of the formation and collimation of jets rely on an externally imposed poloidal magnetic field and disregard any field produced in the disc. Our aim here is to study outflows in connection with dynamo-generated magnetic fields. We discuss parameters of young stellar objects in our estimates, but the model also applies to systems containing a compact central object after rescaling and possibly other modifications such as the appropriate choice of the gravitational potential.

Extensive numerical studies of collimated disc winds have been performed using several types of model. Uchida \& Shibata (1985), Matsumoto et al. (1996) and Kudoh et al. (1998) consider an ideal MHD model of an accretion disc embedded in a non-rotating corona, permeated by an external magnetic field initially aligned with the disc rotation axis. Intense accretion develops in the disc (Stone \& Norman 1994),

Send offprint requests to: $\mathrm{B}$. von Rekowski, e-mail: Brigitta.vonRekowski@astro.uu.se

* Now at: Department of Astronomy \& Space Physics, Uppsala University, Box 515, 75120 Uppsala, Sweden.

$\star \star$ NORDITA, Blegdamsvej 17, 2100 Copenhagen $\varnothing$, Denmark.

$\star \star \star$ Kiepenheuer-Institute für Sonnenphysik, Schöneckstr. 6, 79104 Freiburg, Germany. accompanied by a centrifugally driven outflow. The wind is eventually collimated by toroidal magnetic field produced in the corona by winding up the poloidal field.

Bell (1994), Bell \& Lucek (1995) and Lucek \& Bell (1996, 1997) use two- and three-dimensional ideal MHD models, with a polytropic equation of state, to study the formation and stability of pressure driven jets collimated by poloidal magnetic field, which has a minimum inside the jet. The general structure of thermally driven disc winds was studied analytically by, e.g., Fukue (1989) and Takahara et al. (1989).

Another type of model was developed by Ustyugova et al. (1995), Romanova et al. (1997, 1998), Ouyed et al. (1997) and Ouyed \& Pudritz (1997a, 1997b, 1999) who consider ideal MHD in a polytropic corona permeated by an external poloidal magnetic field and subsume the physics of the accretion disc into boundary conditions at the base of the corona. The disc is assumed to be in Keplerian rotation (with any accretion neglected). The corona is non-rotating initially, and the system is driven by the injection of matter through the boundary that represents the disc surface. These models develop a steady (or at least statistically steady) state consistent with the analytical models by Blandford \& Payne (1982), showing a magnetocentrifugal wind collimated by toroidal magnetic field, which again is produced in the corona by the vertical shear in the angular velocity. Three-dimensional simulations suggest that the resulting collimated outflow does not break due to the kink instability (Ouyed et al. 2003; Thomsen \& Nordlund 2003).

It is not quite clear how strong the external magnetic field in accretion discs can be. Dragging of an external field from large radii in a viscous disc requires that magnetic diffusivity is much smaller than the kinetic one (Lubow et al. 1994), i.e. that the magnetic Prandtl number is significantly larger than unity which would be difficult to explain in a turbulent disc 
(Heyvaerts et al. 1996). This argument neglects, however, the effect of magnetic torques which could produce significant field line dragging even when the magnetic Prandtl number is of order unity (Shalybkov \& Rüdiger 2000). On the other hand, the efficiency of trapping an external magnetic field at initial stages of the disc formation is questionable because only a small fraction of the external flux can be retained if the density contrast between the disc and the surrounding medium is large. In that case the magnetic field will be strongly bent and reconnection will remove most of the flux from the disc. Furthermore, the ionization fraction is probably too small in the inner disc to ensure sufficient coupling between the gas and the magnetic field (Fromang et al. 2002). For these reasons it seems appropriate to explore whether or not magnetic fields generated by a dynamo within the disc can produce an outflow with realistic properties.

The models of Ustyugova et al. (1995) and Ouyed \& Pudritz (1997a, 1997b, 1999) have a distributed mass source and imposed poloidal velocity at the (unresolved) disc surface. As a result, their system develops a persistent outflow which is not just a transient. On the other hand, the models of Matsumoto et al. (1996) resolve the disc, but have no mass replenishment to compensate losses via the outflow and accretion, and so the disc disappears with time. Our model is an attempt to combine advantages of both of these approaches and also to add dynamo action in the disc. Instead of a rigidly prescribed mass injection, we allow for self-regulatory replenishment of matter within a resolved disc. Instead of prescribing poloidal velocity at the disc surface, we resolve the disc and prescribe an entropy contrast between the disc and the corona, leaving more freedom for the system. Such an increase of entropy with height is only natural to expect for a disc surrounded by a hot corona, and we parameterize the coronal heating by a (fixed) entropy contrast. We further add self-sustained, intrinsic magnetic field to our system, as opposed to an external field used in the other models. Since our model goes beyond ideal MHD, the magnetic field must be maintained against decay. A simple form of mean-field dynamo action is included for this purpose.

Like in the model of Ouyed \& Pudritz (1997a, 1997b, 1999), the hot, pressure supported corona does not rotate initially. The disc is cool and is therefore centrifugally supported, so its rotation is nearly Keplerian. The corresponding steady-state solution is used as our initial condition. This solution is, however, unstable because of the vertical shear in the angular velocity between the disc and the corona (Urpin $\&$ Brandenburg 1998). Angular momentum transfer by viscous and magnetic stresses also leads to a departure from the initial state. As a result, a meridional flow develops, which exchanges matter between the corona and the disc surface layers. Mass losses through the disc surface and to the accreting central object are then replenished in the disc where we allow local mass production whenever and wherever the density decreases below a reference value. Matter is heated as it moves into the corona; this leads to an increase in pressure which drives the wind. Another efficient driver of the outflow in our model is magneto-centrifugal acceleration. A strong toroidal magnetic field produced by the dynamo in the disc is advected into the corona and contributes to confining the wind.

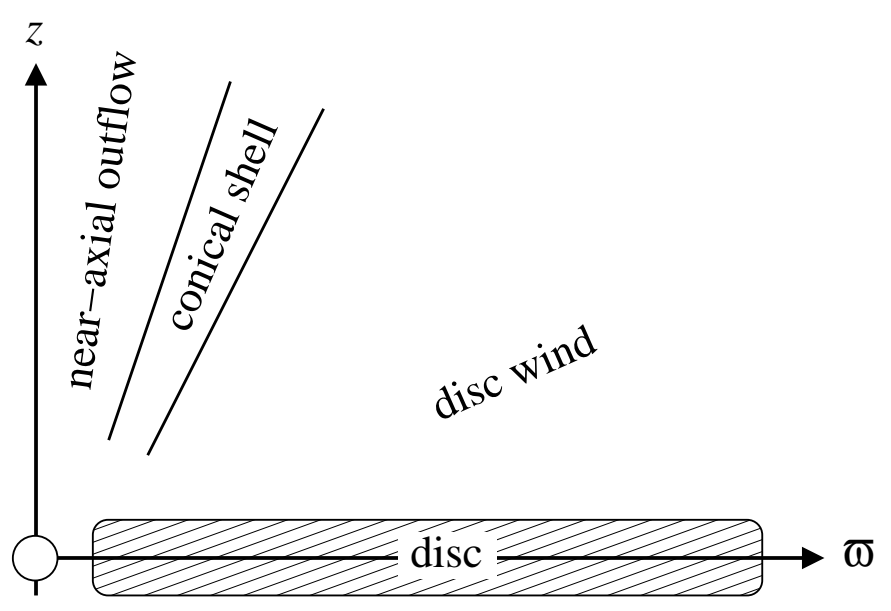

Fig. 1. General structure of the outflows typically obtained in the magnetic runs with mass sink presented in this paper. The cool, dense disc emits (a) a hardly collimated, thermally driven wind (slow, hot, dense, magnetized, rotating), (b) a magneto-centrifugally driven outflow in a conical shell (faster, cooler, less dense, magnetized, quickly rotating), and (c) a thermally driven outflow near the axis (slow, hot, dense, weakly magnetized and weakly rotating).

Altogether, our model contains many features included in a range of earlier models. For example, the disc is essentially resistive to admit dynamo action, whereas magnetic diffusion turns out to be relatively unimportant in the corona, similarly to the models of, e.g., Wardle \& Königl (1993), Ferreira \& Pelletier (1995) and Casse \& Ferreira (2000a, 2000b). The synthetic nature of the outflow, driven by both pressure forces and magneto-centrifugally, is characteristic of the self-similar solutions of Ferreira \& Pelletier (1995) and Casse \& Ferreira (2000b). The latter authors also stress the rôle of the vertical entropy gradient in enhancing the mass flux in the outflow and prescribe a vertical profile of the entropy production rate. Structured outflows outwardly similar to our results have been discussed by Krasnopolsky et al. (1999) and Goodson et al. (1997). However, in the former paper the structure in their solutions is due to the boundary conditions imposed on the disc surface, and in the latter model the structure is due to the interaction between the stellar magnetic field and the disc under the presence of differential rotation between star and disc. On the contrary, the structure in our model results from the dominance of different driving mechanisms in the inner and outer parts of the disc.

While we had initially expected to find collimated outflows similar to those reported by Ouyed \& Pudritz (1997a, 1997b), the outflow patterns we obtained turned out to be of quite different nature. However, a significant difference in our model is the resolved disc, which is necessary to model dynamo action in the disc, and the relatively small extension into the corona, where collimation is not yet expected. Figure 1 shows a sketch of the overall, multi-component structure of the outflows in the presence of magnetic fields and mass sink, which needs to be compared to the individual figures in the rest of the paper.

The plan of the paper is as follows. We introduce our model in Sect. 2, and then consider a range of parameters in Sect. 3 
to clarify and illustrate the main physical effects. In Sect. 4 we explore the parameter space, and our conclusions are presented in Sect. 5.

\section{The model}

\subsection{Basic equations}

Our intention is to make our model as simple as possible and to avoid detailed modelling of mass supply to the accretion disc, which occurs differently in different accreting systems. For example, matter enters the accretion disc at large radii in a restricted range of azimuthal angles in binary systems with Roche lobe overflow. On the other hand, matter supply can be more uniform in both azimuth and radius in active galactic nuclei. Being interested in other aspects of accretion physics, we prefer to avoid detailed modelling of these processes. Instead, similarly to the model of Ouyed \& Pudritz (1997a, 1997b), we inject matter into the system, but an important difference is that we introduce a self-regulating mass source in the disc. We also allow for a mass sink at the centre to model accretion onto the central object (star). With these two effects included, the continuity equation becomes

$\frac{\partial \varrho}{\partial t}+\nabla \cdot(\varrho \boldsymbol{u})=q_{\varrho}^{\mathrm{disc}}+q_{\varrho}^{\mathrm{star}} \equiv \dot{\varrho}$,

where $\boldsymbol{u}$ is the velocity field and $\varrho$ is the gas density. The mass source, $q_{\varrho}^{\text {disc }}$, is localized in the disc (excluding the star) and turns on once the local density drops below a reference value $\varrho_{0}$ (chosen to be equal to the initial density, see Sect. 2.4), i.e.

$q_{\varrho}^{\mathrm{disc}}=\frac{\xi_{\mathrm{disc}}(\boldsymbol{r})-\xi_{\mathrm{star}}(\boldsymbol{r})}{\tau_{\mathrm{disc}}}\left(\varrho_{0}-\varrho\right)_{+}$,

where the subscript "+" indicates that only positive values are used, i.e.

$x_{+}= \begin{cases}x & \text { if } x>0 \\ 0 & \text { otherwise. }\end{cases}$

This means that matter is injected only if $\varrho<\varrho_{0}$, and that the strength of the mass source is proportional to the gas density deficit. Throughout this work we use cylindrical polar coordinates $(\varpi, \varphi, z)$, assuming axisymmetry. The shapes of the disc and the central object are specified with the profiles $\xi_{\text {disc }}$ and $\xi_{\text {star via }}$

$\xi_{\text {disc }}(\varpi, z)=\Theta\left(\varpi_{0}-\varpi, d\right) \Theta\left(z_{0}-|z|, d\right)$,

$\xi_{\text {star }}(\varpi, z)=\Theta\left(r_{0}-r, d\right)$.

Here, $\Theta(x, d)$ is a smoothed Heaviside step function with a smoothing half-width $d$ set to 8 grid zones in $\xi_{\text {disc }}$ and $d=r_{0}$ in $\xi_{\text {star }} ; r=\sqrt{\varpi^{2}+z^{2}}$ is the spherical radius and $r_{0}$ is the stellar radius introduced in Eq. (23); $\varpi_{0}$ and $z_{0}$ are the disc outer radius and disc half-thickness, respectively. So, $\xi_{\text {disc }}$ is equal to unity at the disc midplane and vanishes in the corona, whereas $\xi_{\text {star }}$ is unity at the centre of the central object and vanishes outside the star. Note that $\xi_{\text {disc }}-\xi_{\text {star }} \geq 0$ everywhere, since $\xi_{\text {disc }}>0$ ) all the way to the origin, $z=\varpi=0$.
In Eq. (2), $\tau_{\text {disc }}$ is a response time which is chosen to be significantly shorter than the time scale of the depletion processes, which is equivalent to the time scale of mass replenishment in the disc, $M_{\text {disc }} / \dot{M}_{\text {source }}$ (cf. Sect. 3.4), to avoid unphysical influences of the mass source. We do not fix the distribution and magnitude of $q_{\varrho}^{\text {disc }}$ beforehand, but the system adjusts itself such as to prevent the disc from disappearing.

The self-regulating mass sink at the position of the central star is modelled in a similar manner,

$q_{\varrho}^{\text {star }}=-\frac{\xi_{\text {star }}(\boldsymbol{r})}{\tau_{\text {star }}}\left(\varrho-\varrho_{0}\right)_{+}$,

where $\xi_{\text {star }}$ is defined in Eq. (5) and $\tau_{\text {star }}$ is a central accretion time scale that controls the efficiency of the sink. We discuss physically meaningful values of $\tau_{\text {star }}$ in Sect. 2.5.

Apart from the continuity Eq. (1), the mass source also appears in the Navier-Stokes equation, unless matter is always injected with the ambient velocity of the gas. In that case, however, a runaway instability can occur: if matter is already slower than Keplerian, it falls inward, and so does the newly injected matter. This enhances the need for even more mass injection. A similar argument applies also if matter is rotating faster than Keplerian. This is why we inject matter at the Keplerian speed. This leads to an extra term in the Navier-Stokes equation, $\left(\boldsymbol{u}_{\mathrm{K}}-\boldsymbol{u}\right) q_{\varrho}^{\text {disc }}$, which would only be absent if the gas were rotating at the Keplerian speed. Thus, the Navier-Stokes equation takes the form

$\frac{\mathrm{D} \boldsymbol{u}}{\mathrm{D} t}=-\frac{1}{\varrho} \boldsymbol{\nabla} p-\boldsymbol{\nabla} \Phi+\frac{1}{\varrho}\left[\boldsymbol{F}+\left(\boldsymbol{u}_{\mathrm{K}}-\boldsymbol{u}\right) q_{\varrho}^{\mathrm{disc}}\right]$,

where $\mathrm{D} / \mathrm{D} t=\partial / \partial t+\boldsymbol{u} \cdot \boldsymbol{\nabla}$ is the advective derivative, $p$ is the gas pressure, $\Phi$ is the gravitational potential, $\boldsymbol{F}=\boldsymbol{J} \times \boldsymbol{B}+\boldsymbol{\nabla} \cdot \boldsymbol{\tau}$ is the sum of the Lorentz and viscous forces, $\boldsymbol{J}=\boldsymbol{\nabla} \times \boldsymbol{B} / \mu_{0}$ is the current density due to the (mean) magnetic field $\boldsymbol{B}, \mu_{0}$ is the magnetic permeability, and $\tau$ is the viscous stress tensor,

$\tau_{i j}=\varrho v\left(\partial u_{i} / \partial x_{j}+\partial u_{j} / \partial x_{i}\right)$.

Here, $v$ is the kinematic viscosity, which has been subdivided into three contributions,

$v=v_{\mathrm{t}}+v_{\mathrm{adv}}+v_{\text {shock }}$.

The first term is a turbulent (Shakura-Sunyaev) viscosity in the disc,

$v_{\mathrm{t}}=\alpha_{\mathrm{SS}} c_{\mathrm{s}} z_{0} \xi_{\mathrm{disc}}(\boldsymbol{r})$

where $c_{\mathrm{s}}=(\gamma p / \varrho)^{1 / 2}$ is the sound speed and $\gamma$ is the ratio of specific heats. The second term is an artificial advection viscosity,

$\nu_{\mathrm{adv}}=c_{v}^{\mathrm{adv}} \delta x\left(\boldsymbol{u}_{\mathrm{pol}}^{2}+c_{\mathrm{s}}^{2}+v_{\mathrm{A}, \mathrm{pol}}^{2}\right)^{1 / 2}$,

which is required to stabilize rapidly moving patterns. Here, $\delta x=\min (\delta \varpi, \delta z)$ is the mesh size, and $c_{v}^{\text {adv }}$ is a constant specified in Sect. 2.5. In Eq. (11) we have only used the poloidal velocity, $\boldsymbol{u}_{\mathrm{pol}}$, and the Alfvén speed due to the poloidal magnetic field, $v_{\mathrm{A}, \mathrm{pol}}=\left(\boldsymbol{B}_{\mathrm{pol}}^{2} / \varrho \mu_{0}\right)^{1 / 2}$, because in an axisymmetric calculation advection in the $\varphi$-direction is unimportant. Finally,

$v_{\text {shock }}=c_{v}^{\text {shock }} \delta x^{2}(-\boldsymbol{\nabla} \cdot \boldsymbol{u})_{+}$ 
is an artificial shock viscosity, with $c_{v}^{\text {shock }}$ a constant specified in Sect. 2.5. Note that $v_{\mathrm{adv}}$ and $v_{\text {shock }}$ are needed for numerical reasons; they tend to zero for increasing resolution, $\delta x \rightarrow 0$.

We assume that the magnetic field in the disc is generated by a standard $\alpha^{2} \Omega$-dynamo (e.g., Krause \& Rädler 1980), which implies an extra electromotive force, $\alpha \boldsymbol{B}$, in the induction equation for the mean magnetic field, $\boldsymbol{B}$. To ensure that $\boldsymbol{B}$ is solenoidal, we solve the induction equation in terms of the vector potential $\boldsymbol{A}$,

$\frac{\partial \boldsymbol{A}}{\partial t}=\boldsymbol{u} \times \boldsymbol{B}+\alpha \boldsymbol{B}-\eta \mu_{0} \boldsymbol{J}$,

where $\boldsymbol{B}=\boldsymbol{\nabla} \times \boldsymbol{A}$, and $\eta$ is the magnetic diffusivity.

Since $\alpha(\boldsymbol{r})$ has to be antisymmetric about the midplane and vanishing outside the disc, we adopt the form

$\alpha=\alpha_{0} \frac{z}{z_{0}} \frac{\xi_{\mathrm{disc}}(\boldsymbol{r})}{1+v_{\mathrm{A}}^{2} / v_{0}^{2}}$,

where $v_{\mathrm{A}}$ is the Alfvén speed based on the total magnetic field, and $\alpha_{0}$ and $v_{0}$ are parameters that control the intensity of dynamo action and the field strength in the disc, respectively. The $\alpha$-effect has been truncated near the axis, so that $\alpha=0$ for $\varpi \leq 0.2$. For the magnetic diffusivity we assume

$\eta=\eta_{0}+\eta_{\mathrm{t}}$,

where $\eta_{0}$ is a uniform background diffusivity and the turbulent part, $\eta_{\mathrm{t}}=\eta_{\mathrm{t} 0} \xi_{\text {disc }}(\boldsymbol{r})$, vanishes outside the disc. Thus, magnetic diffusivity in the corona is smaller than in the disc.

Depending on the sign of $\alpha$ and the vertical distribution of $\eta$, the dynamo can generate magnetic fields of either dipolar or quadrupolar symmetry. We shall discuss both types of geometry.

\subsection{Implementation of a cool disc embedded in a hot corona}

Protostellar systems are known to be strong X-ray sources (see, e.g., Glassgold et al. 2000; Feigelson \& Montmerle 1999; Grosso et al. 2000). The X-ray emission is generally attributed to coronae of disc-star systems, plausibly heated by small scale magnetic reconnection events (Galeev et al. 1979), for example in the form of nanoflares that are caused by slow footpoint motions (Parker 1994). Heating of disc coronae by fluctuating magnetic fields is indeed quite natural if one accepts that the disc turbulence is caused by the magneto-rotational instability. Estimates for the coronal temperatures of YSOs range from $10^{6} \mathrm{~K}$ to $10^{8} \mathrm{~K}$ (see, e.g., Feigelson \& Montmerle 1999). For the base of the disc corona, temperatures of at least $8 \times 10^{3}$ are to be expected in order to explain the observed mass loss rates (Kwan \& Tademaru 1995; Kwan 1997). The discs, on the other hand, have typical temperatures of a few $10^{3} \mathrm{~K}$ (e.g., Papaloizou \& Terquem 1999).

A simple way to implement a dense, relatively cool disc embedded in a rarefied, hot corona without modelling the detailed physics of coronal heating is to prescribe the distribution of specific entropy, $s(\boldsymbol{r})$, such that $s$ is smaller within the disc and larger in the corona. For a perfect gas this implies $p=K \varrho^{\gamma}$ (in a dimensionless form), where $K=\mathrm{e}^{s / c_{\mathrm{v}}}$ is a function of position (here $p$ and $\varrho$ are gas pressure and density, $\gamma=c_{\mathrm{p}} / c_{\mathrm{v}}$, and $c_{\mathrm{p}}$ and $c_{\mathrm{v}}$ are the specific heats at constant pressure and constant volume, respectively).

We prescribe the polytrope parameter $K$ to be unity in the corona and smaller in the disc, so we put

$[K(\boldsymbol{r})]^{1 / \gamma}=\mathrm{e}^{s / c_{\mathrm{p}}}=1-(1-\beta) \xi_{\text {disc }}(\boldsymbol{r})$,

where $0<\beta<1$ is a free parameter with $\beta=\mathrm{e}^{-\Delta s / c_{\mathrm{p}}}$, that controls the entropy contrast, $\Delta s>0$, between corona and disc. We consider values of $\beta$ between 0.1 and 0.005 , which yields an entropy contrast, $\Delta s / c_{\mathrm{p}}$, between 2.3 and 5.3. The temperature ratio between disc and corona is roughly $\beta$; see Eq. (26). Assuming pressure equilibrium between disc and corona, and $p \propto \rho T$ for a perfect gas, the corresponding density ratio is $\beta^{-1}$.

\subsection{Formulation in terms of potential enthalpy}

In the present case it is advantageous to use the potential enthalpy,

$H=h+\Phi$,

as a variable. Here, $h$ is the specific enthalpy, $h=c_{\mathrm{v}} T+p / \varrho=$ $c_{\mathrm{p}} T$ for a perfect gas (with constant specific heats), and $T$ is temperature. Therefore, specific enthalpy $h$ is related to $p$ and $\varrho$ via $h=\gamma(\gamma-1)^{-1} p / \varrho$. Specific entropy $s$ is related to $p$ and $\varrho$ (up to an additive constant) through $s=c_{\mathrm{v}} \ln p-c_{\mathrm{p}} \ln \varrho$ for a perfect gas. We have therefore

$\frac{\mathrm{D} \ln h}{\mathrm{D} t}=\frac{\mathrm{D} \ln p}{\mathrm{D} t}-\frac{\mathrm{D} \ln \varrho}{\mathrm{D} t}=(\gamma-1) \frac{\mathrm{D} \ln \varrho}{\mathrm{D} t}+\frac{\gamma}{c_{\mathrm{p}}} \frac{\mathrm{D} s}{\mathrm{D} t}$.

Since $s$ is independent of time, $\mathrm{D} s / \mathrm{D} t=\boldsymbol{u} \cdot \boldsymbol{\nabla} s$. Together with Eq. (1), this yields an evolution equation for $h$, which can be written in terms of $H$ as

$\frac{\mathrm{D} H}{\mathrm{D} t}=\boldsymbol{u} \cdot \boldsymbol{\nabla} \Phi+(\gamma-1) h\left(\frac{\dot{\underline{Q}}}{\varrho}-\boldsymbol{\nabla} \cdot \boldsymbol{u}\right)+\frac{\gamma h}{c_{\mathrm{p}}} \boldsymbol{u} \cdot \boldsymbol{\nabla} s$.

In the following we solve Eq. (19) instead of Eq. (1). In terms of $h$, density and sound speed are given by

$\varrho^{\gamma-1}=\frac{\gamma-1}{\gamma} \frac{h}{K}, \quad c_{\mathrm{s}}^{2}=(\gamma-1) h=\gamma \frac{p}{\varrho}$.

It proved necessary to include an artificial diffusion term in Eq. (19) with a diffusion coefficient proportional to $v$.

The first law of thermodynamics allows us to express the pressure gradient in terms of $h$ and $s$,

$-\frac{1}{\varrho} \nabla p=-\nabla h+T \nabla s$,

and with $T=h / c_{\mathrm{p}}$ we obtain

$\frac{\mathrm{D} \boldsymbol{u}}{\mathrm{D} t}=-\boldsymbol{\nabla} H+h \boldsymbol{\nabla} s / c_{\mathrm{p}}+\frac{1}{\varrho}\left[\boldsymbol{F}+\left(\boldsymbol{u}_{\mathrm{K}}-\boldsymbol{u}\right) q_{\varrho}^{\mathrm{disc}}\right]$,

which now replaces Eq. (7).

We use a softened, spherically symmetric gravitational potential of the form

$\Phi=-G M_{*}\left(r^{n}+r_{0}^{n}\right)^{-1 / n}$, 
where $G$ is the gravitational constant, $M_{*}$ is the mass of the central object, $r$ is the spherical radius, $r_{0}$ is the softening radius, and $n=5$; tentatively, $r_{0}$ can be identified with the stellar radius.

\subsection{The initial state}

Our initial state is the hydrostatic equilibrium obtained by solving, for $h$, the vertical balance equation obtained from Eq. (22),

$-\frac{\partial}{\partial z}(h+\Phi)+h \frac{\partial s / c_{\mathrm{p}}}{\partial z}=0$

from large $z$ (where $h+\Phi=0$ ) down to $z=0$. The initial density distribution $\varrho_{0}$ is then obtained using Eq. (20); it decreases monotonically with both $z$ and $\varpi$ in the equilibrium state.

The initial rotation velocity, $u_{\varphi 0}$, follows from the radial balance equation,

$-\frac{u_{\varphi 0}^{2}}{\varpi}=-\frac{\partial}{\partial \varpi}(h+\Phi)+h \frac{\partial s / c_{\mathrm{p}}}{\partial \varpi}$.

In the disc, $h=c_{\mathrm{p}} T$ is small, so $u_{\varphi 0}$ is close to the Keplerian velocity, while the corona does not rotate initially, and so is supported by the pressure gradient.

As a rough estimate, the value of $h$ in the midplane of the disc is

$h_{\mathrm{disc}} \approx \beta h_{\text {corona }} \equiv \beta G M_{*} / \varpi$,

as can be seen by integrating Eq. (24), ignoring the $\partial \Phi / \partial z$ term. Thus, the initial toroidal velocity in the midplane can be obtained from Eq. (25) using Eq. (26) and recalling that $\partial s / \partial \varpi=0$ in the midplane, which gives

$u_{\varphi 0} \approx \sqrt{(1-\beta) G M_{*} / \varpi}=\sqrt{1-\beta} \boldsymbol{u}_{\mathrm{K}}$.

For $\beta=0.1$, for example, the toroidal velocity is within $5 \%$ of the Keplerian velocity.

\subsection{Dimensionless variables and choice of parameters}

Our model is scale invariant and can therefore be applied to various astrophysical objects. We consider here the range of parameters typical of protostellar discs, for which a typical surface density is $\Sigma_{0} \approx 1 \mathrm{~g} \mathrm{~cm}^{-2}$. A typical coronal sound speed is $c_{\mathrm{s} 0} \approx 10^{2} \mathrm{~km} \mathrm{~s}^{-1}$, which corresponds to a temperature of $T \approx 4 \times 10^{5} \mathrm{~K}$. This allows us to fix relevant units as follows:

$[\Sigma]=\Sigma_{0}, \quad[u]=c_{\mathrm{s} 0}, \quad[x]=G M_{*} c_{\mathrm{s} 0}^{-2}, \quad[s]=c_{\mathrm{p}}$.

With $M_{*}=1 M_{\odot}$ we have $[x] \approx 0.1 \mathrm{AU}$, and $[t]=[x] /[u] \approx$ 1.5 day. The unit for density is $[\varrho]=[\Sigma] /[x] \approx 7 \times 10^{-13} \mathrm{~g} \mathrm{~cm}^{-3}$, the unit for the mass accretion rate is $[\dot{M}]=[\Sigma][u][x] \approx$ $2 \times 10^{-7} M_{\odot} \mathrm{yr}^{-1}$, and the unit for the magnetic field is $[B]=$ $[u]\left(\mu_{0}[\varrho]\right)^{1 / 2} \approx 30 \mathrm{G}$.

Since $[h]=[u]^{2}$, the dimensionless value $h=1$ corresponds to $10^{14} \mathrm{~cm}^{2} \mathrm{~s}^{-2}$. With a mean specific weight $\mu=0.6$, the universal gas constant $\mathcal{R}=8.3 \times 10^{7} \mathrm{~cm}^{2} \mathrm{~s}^{-2} \mathrm{~K}^{-1}$ and $\gamma=5 / 3$, we have

$c_{\mathrm{p}}=\frac{\gamma}{\gamma-1} \frac{\mathcal{R}}{\mu} \approx 3.5 \times 10^{8} \mathrm{~cm}^{2} \mathrm{~s}^{-2} \mathrm{~K}^{-1}$.
Therefore, $h=1$ corresponds to $T=[u]^{2} /[s]=[h] / c_{\mathrm{p}} \approx$ $3 \times 10^{5} \mathrm{~K}$. Using $h=-\Phi$ in the corona, this corresponds to a temperature of $3 \times 10^{5} \mathrm{~K}$ at $r=[x] \equiv 0.1 \mathrm{AU}$.

We choose $\beta$ between 0.1 and 0.005 , corresponding to a typical disc temperature (in the model) of $3 \times 10^{4} \mathrm{~K}$ to $1.5 \times 10^{3} \mathrm{~K}$; see Eq. (26); real protostellar discs have typical temperatures of about a few thousand Kelvin (see Sect. 2.2).

In our models, we use $\varpi_{0}=1.5$ for the disc outer radius, $z_{0}=0.15$ for the disc semi-thickness and $r_{0}=0.05$ for the softening (stellar) radius. The disc aspect ratio is $z_{0} / \varpi_{0}=0.1$. Note that $r_{0}=0.05$ corresponds to $7 \times 10^{10} \mathrm{~cm}$, i.e. one solar radius. Therefore, we shall not reduce it much below this physically meaningful value. Note, however, that smaller values of $r_{0}$ would result in faster outflows (Ouyed \& Pudritz 1999).

Furthermore, $c_{v}^{\text {adv }}=0.02$ and $c_{v}^{\text {shock }}=1.2$. We vary the value of $\alpha_{\mathrm{SS}}$ between 0.003 and 0.007 .

The mean-field dynamo is characterized by the parameters $\left|\alpha_{0}\right|=0.3, v_{0}=c_{\mathrm{s}}, \eta_{\mathrm{t} 0}=10^{-3}$ and $\eta_{0}=5 \times 10^{-4}$. The total magnetic diffusivity in the disc is therefore $\eta_{\mathrm{T} 0}=\eta_{\mathrm{t} 0}+\eta_{0}=0.0015$. In terms of the usual Shakura-Sunyaev viscosity parameter, this corresponds to

$\alpha_{\mathrm{SS}}^{(\eta)} \equiv \frac{\eta_{\mathrm{T} 0}}{c_{\mathrm{S}, \mathrm{disc}} z_{0}} \approx 0.01\left(\frac{\varpi}{\beta}\right)^{1 / 2}\left(\frac{\eta_{\mathrm{T} 0}}{0.0015}\right)\left(\frac{z_{0}}{0.15}\right)^{-1}$,

where we have used $c_{\mathrm{s} \text {,disc }}^{2} \approx \beta c_{\mathrm{s} \text {,corona }}^{2}$ (cf. Eqs. (20) and (26)) and $c_{\mathrm{s}, \text { corona }} \approx \varpi^{-1 / 2}$.

In terms of the usual nondimensional dynamo parameters we have

$\left|C_{\alpha}\right|=\left|\alpha_{0}\right| z_{0} / \eta_{\mathrm{T} 0}=30$

and, for Keplerian rotation,

$C_{\omega}=(\varpi \partial \Omega / \partial \varpi) z_{0}^{2} / \eta_{\mathrm{T} 0}=-22.5 \varpi^{-3 / 2}$,

so that the dynamo number is given by

$|\mathcal{D}|=\left|C_{\alpha} C_{\omega}\right|=675 \varpi^{-3 / 2}$.

Note that the value of the dynamo number expected for accretion discs is given by

$|\mathcal{D}|=\frac{\left|\alpha_{0} \varpi \partial \Omega / \partial \varpi\right| z_{0}^{3}}{\eta_{\mathrm{T} 0}^{2}} \lesssim \frac{3}{2}\left(\alpha_{\mathrm{SS}}^{(\eta)}\right)^{-2}$

for $\eta_{\mathrm{T} 0}=\alpha_{\mathrm{SS}}^{(\eta)} c_{\mathrm{s}, \mathrm{disc}} z_{0},\left|\alpha_{0}\right| \lesssim c_{\mathrm{s} \text {,disc }}$ and $c_{\mathrm{s}, \mathrm{disc}}=\Omega z_{0}$. As follows from Eqs. (30)-(33), for Keplerian rotation,

$\frac{2}{3}|\mathcal{D}|\left(\alpha_{\mathrm{SS}}^{(\eta)}\right)^{2}=\left|\alpha_{0}\right| z_{0} \beta^{-1} \varpi^{-1 / 2}$.

(Note that this expression is independent of $\eta_{\mathrm{T} 0}$.) For our choice of parameters of $\left|\alpha_{0}\right|=0.3$ and $z_{0}=0.15$, expression (35) is $\lesssim 1$ if $\varpi \gtrsim 0.002 \beta^{-2}=0.2$ for $\beta=0.1$, which corresponds to the truncation radius of the $\alpha$-effect. Therefore, our choice of parameters is consistent with the constraint (34).

We take $\gamma=5 / 3, \tau_{\text {disc }}=0.1$, and consider two values of $\tau_{\text {star }}$. For $\tau_{\text {star }} \rightarrow \infty$, the mass sink at the central object is suppressed, whereas $\tau_{\text {star }} \rightarrow 0$ implies instantaneous accretion of any extra matter (relative to the hydrostatic equilibrium) by the central object. A realistic lower limit for $\tau_{\text {star }}$ can be estimated 
as $\tau_{\text {star }} \gtrsim r_{0} / v_{\mathrm{ff}} \approx 0.008$, where $v_{\mathrm{ff}}$ is the free fall velocity (given by $\frac{1}{2} v_{\mathrm{ff}}^{2}=G M_{*} / r_{0}$ in dimensional quantities). In most cases we used $\tau_{\text {star }}=0.01$, but we also tried an even smaller value of $\tau_{\text {star }}=0.005$ and obtained very similar results. A finite value of $\tau_{\text {star }}$ implies that matter is not instantaneously absorbed by the sink. Therefore, some matter can leave the sink if it moves so fast that its residence time in the sink is shorter than $\tau_{\text {star. As }}$ can be seen below, a small (negligible) fraction of mass does indeed escape from the sink.

Computations have been carried out in domains ranging from $(\varpi, z) \in[0,2] \times[-1,1]$ to $[0,8] \times[-2,30]$, but the results are hardly different in the overlapping parts of the domains. In our standard computational box, $\delta \varpi=\delta z=0.01$ and in the case of the larger computational domain $\delta \varpi=\delta z=0.02$.

\subsection{Numerical method and boundary conditions}

We use third order Runge-Kutta time-stepping and a sixth order finite-difference scheme in space. Details and test calculations are discussed by Brandenburg (2003).

On the outer boundaries, the induction equation is evolved using one-sided derivatives (open boundary conditions). The normal velocity component has zero derivative normal to the boundary, but the velocity is required to be always directed outwards. The tangential velocity components and potential enthalpy on the boundaries are similarly obtained from the next two interior points away from the boundary. Tests show that the presence of the boundaries does not affect the flow inside the computational domain. Regularity conditions are adopted on the axis where the $\varpi$ and $\varphi$ components of vectorial quantities vanish, whereas scalar variables and the $z$ components of vectorial quantities have vanishing radial derivative.

\section{Results}

In this section we discuss a range of models of increasing degree of complexity. We first consider in Sect. 3.1 the simplest model that contains neither magnetic field nor mass sink at the centre to show how the pressure gradient resulting from the (fixed) entropy distribution drives a disc outflow. It is further shown that the outflow is significantly reduced if accretion onto the central object is allowed, but restored again if the disc can generate a large-scale magnetic field (Sect. 3.2). Having thus demonstrated the importance of the large-scale magnetic field in our model, we discuss in Sect. 3.3 its structure, largely controlled by the dynamo action but affected by the outflow. Model parameters used in these sections are not necessarily realistic as we aim to illustrate the general physical nature of our solutions. We present a physically motivated model in Sect. 4 where the set of model parameters is close to that of a standard accretion disc around a protostellar object. The physical nature of our solutions is discussed in Sects. 3.4-3.6.

\subsection{Nonmagnetic outflows}

We illustrate in Fig. 2 results obtained for a model without any magnetic fields and without mass sink. A strong outflow develops even in this case, which is driven mostly by the vertical

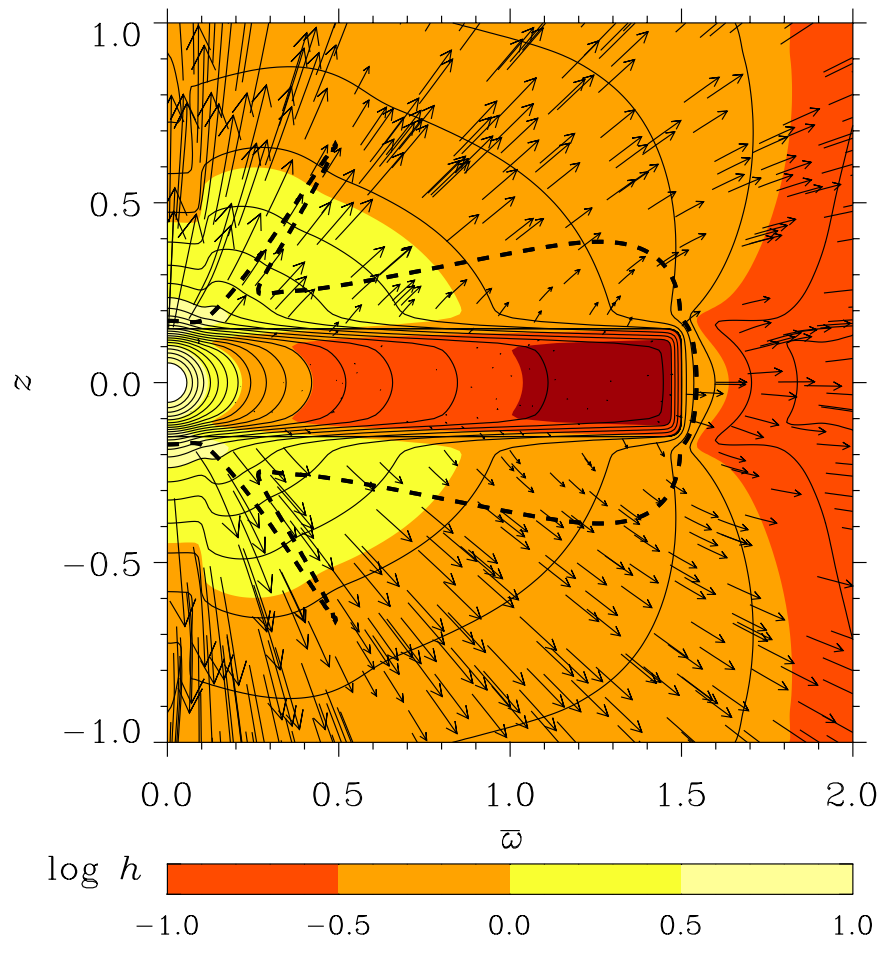

Fig. 2. A nonmagnetic model without mass sink at the centre: velocity vectors and logarithmically spaced density contours (from 0.008 to 6000 , separation factor of 1.6) superimposed on a grey scale representation of $\log _{10} h$ at time $t=223$. Specific enthalpy $h$ is directly proportional to temperature $T$, and $\log _{10} h=(-1,0,1)$ corresponds to $T \approx\left(3 \times 10^{4}, 3 \times 10^{5}, 3 \times 10^{6}\right) \mathrm{K}$. Note hot gas near the central object and in the near corona, and cooler gas in the disc and the far corona. The dashed line shows the sonic surface where the poloidal velocity equals $c_{\mathrm{s}}$. The disc boundary is shown with a thin black line; $\beta=0.1$.

pressure gradient in the transition layer between the disc and the corona, in particular by the term $T \nabla s$ in Eq. (21). The gain in velocity is controlled by the total specific entropy difference between the disc and the corona, but not by the thickness $d$ of the transition layer in the disc profile (4).

The flow is fastest along the rotation axis and within a cone of polar angle of about $30^{\circ}$, where the terminal velocity $u_{z} \approx 3$ is reached. The conical shape of the outflow is partly due to obstruction from the dense disc, making it easier for matter to leave the disc near the axis. Both temperature and density in nonmagnetic runs without mass sink are reduced very close to the axis where the flow speed is highest.

The general flow pattern is sensitive to whether or not matter can accrete onto the central object. We show in Fig. 3 results for the same model as in Fig. 2, but with a mass sink given by Eq. (6) with $\tau_{\text {star }}=0.01$. This can be compared with earlier work on thermally driven winds by Fukue (1989) who also considered polytropic outflows, but the disc was treated as a boundary condition. In Fukue (1989), outflows are driven when the injected energy is above a critical value. The origin of this energy injection may be a hot corona. The critical surface in his model (see the lower panel of his Fig. 2) is quite similar to that found in our simulation (our Fig. 3), although our opening angle was found to be larger than in Fukue's (1989) model. (Below we show, albeit with magnetic fields, that smaller 


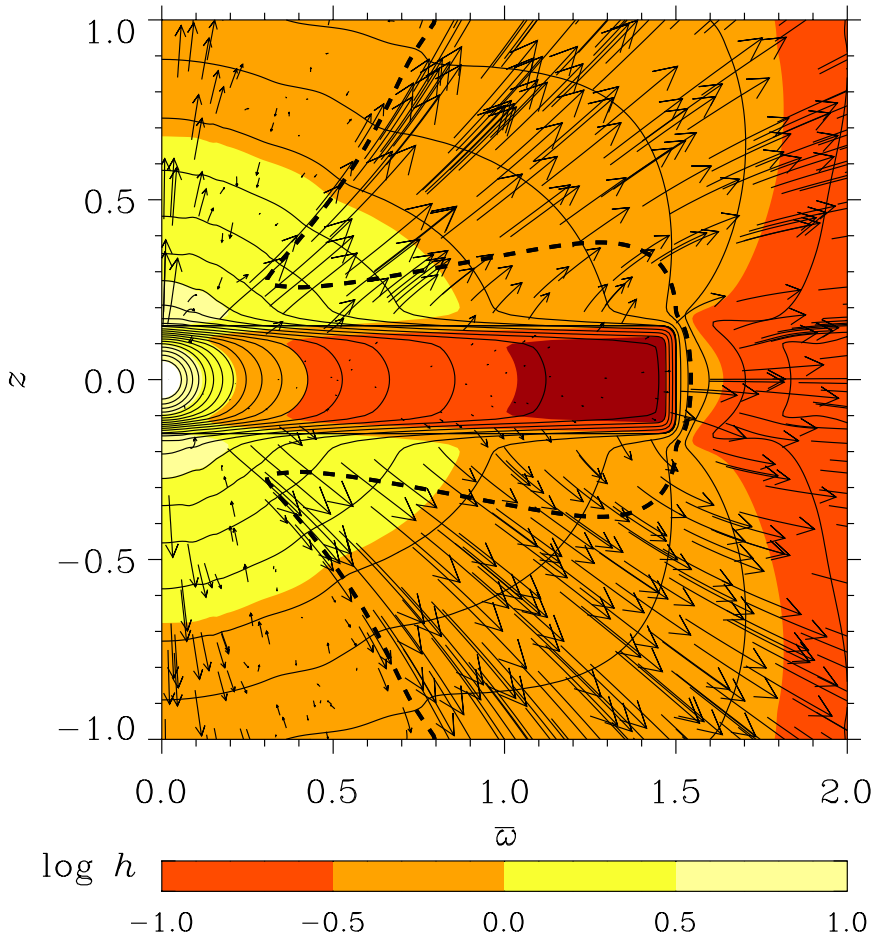

Fig. 3. As in Fig. 2, but with a mass sink at the centre, $\tau_{\text {star }}=0.01$, at time $t=312$. The outflow speed near the axis is strongly reduced in comparison to that in the model without mass sink shown in Fig. 2.

values of $\beta$ do result in smaller opening angles, see Fig. 9, which would then be compatible with the result of Fukue (1989).)

As could be expected, the mass sink hampers the outflow in the cone (but not at $\varpi \gtrsim 0.5$ ). The flow remains very similar to that of Fig. 3 when $\tau_{\text {star }}$ is reduced to 0.005 . Thus, the nonmagnetic outflows are very sensitive to the presence of the central sink. As we show now, magnetized outflows are different in this respect.

\subsection{Magnetized outflows}

In this section we discuss results obtained with magnetic fields, first without mass sink at the centre and then including a sink. We show that the effects of the sink are significantly weaker than in the nonmagnetic case.

A magnetized outflow without the central mass sink, shown in Fig. 4, is similar to that in Fig. 2, but is denser and hotter near the axis, and the high speed cone has a somewhat larger opening angle. In addition, the outflow becomes more structured, with a well pronounced conical shell where temperature and density are smaller than elsewhere (the conical shell reaches $z= \pm 1$ at $\varpi \approx 1.2$ in Fig. 4). Here and in some of the following figures we also show the fast magnetosonic surface with respect to the poloidal field. In Sect. 3.5 we show that this surface is close to the fast magnetosonic surface. As shown in Fig. 5, the outflow becomes faster inside the cone $\left(u_{z} \approx 5\right.$ on the axis). As expected, we find that deeper potential wells, i.e. smaller values of $r_{0}$ in Eq. (5) and (23), result in even faster flows and in larger opening angles.

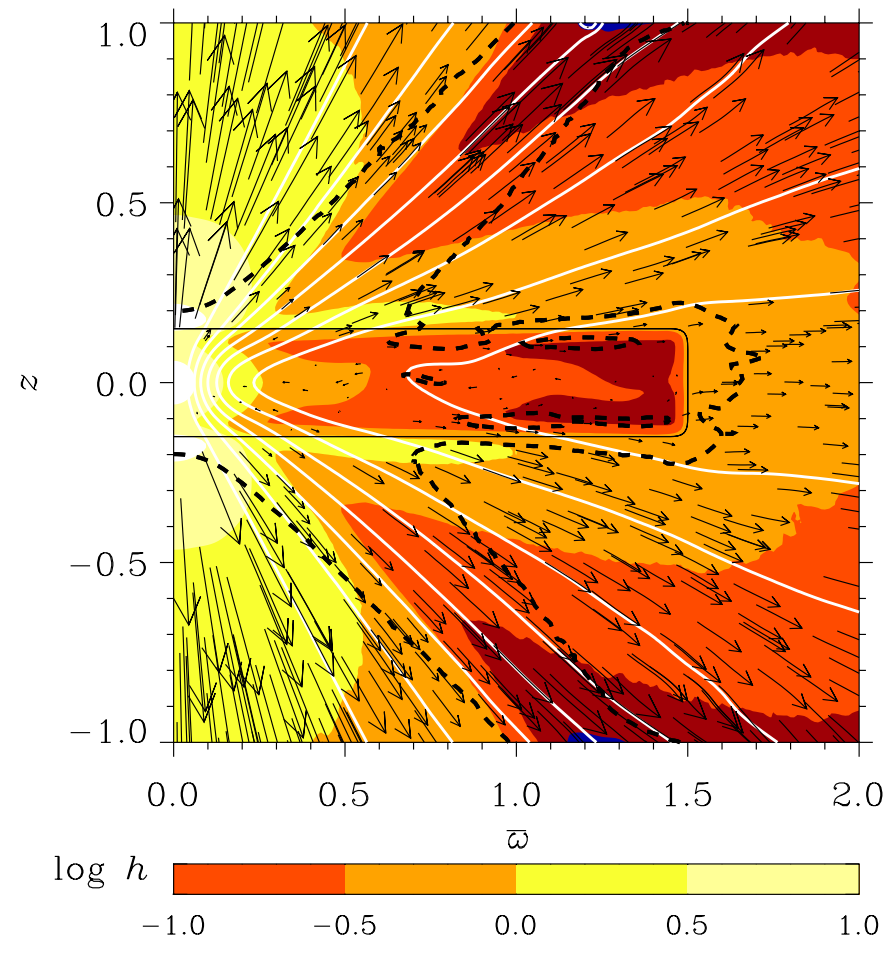

Fig. 4. A magnetic model without mass sink at the centre: velocity vectors and poloidal magnetic field lines (white) superimposed on a grey scale representation of temperature (in terms of $h$ ) for a run with $\alpha_{0}=-0.3$ (resulting in roughly dipolar magnetic symmetry) at time $t=269$. In contrast to the nonmagnetic model of Fig. 2, a conical shell has developed that is cooler and less dense than its exterior. The conical shell intersects $z= \pm 1$ at around $\varpi \approx 1.2$. The dashed line shows the surface where the poloidal velocity equals $\left(c_{\mathrm{s}}^{2}+v_{\mathrm{A}, \text { pol }}^{2}\right)^{1 / 2}$, with $v_{\mathrm{A} \text {,pol }}$ the Alfvén speed from the poloidal magnetic field (fast magnetosonic surface with respect to the poloidal field). The disc boundary is shown with a thin black line; $\beta=0.1$.

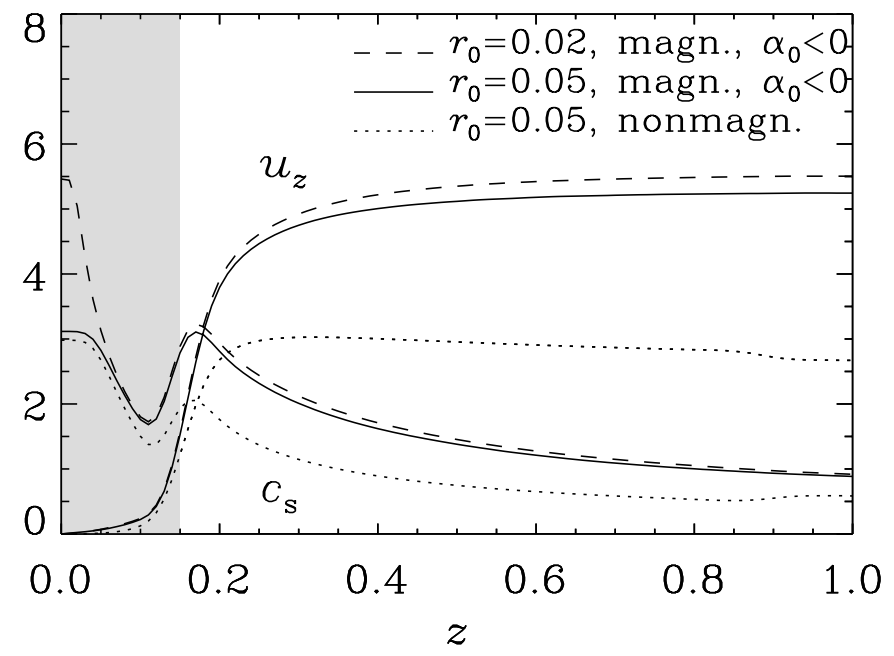

Fig. 5. Profiles of vertical velocity and sound speed along the axis, $\varpi=0$, for the nonmagnetic model of Fig. 2 with smoothing radius $r_{0}=0.05$ (dotted) and two runs with magnetic field, with $r_{0}=0.05$ as in Fig. 4 (solid) and $r_{0}=0.02$ (dashed, $t=162$ ). The shaded area marks the location of the disc. Terminal wind speeds are reached after approximately three disc heights. The presence of a magnetic field and a deeper potential well (smaller $r_{0}$ ) both result in faster outflows. The models shown here have no mass sink at the centre and $\beta=0.1$. 


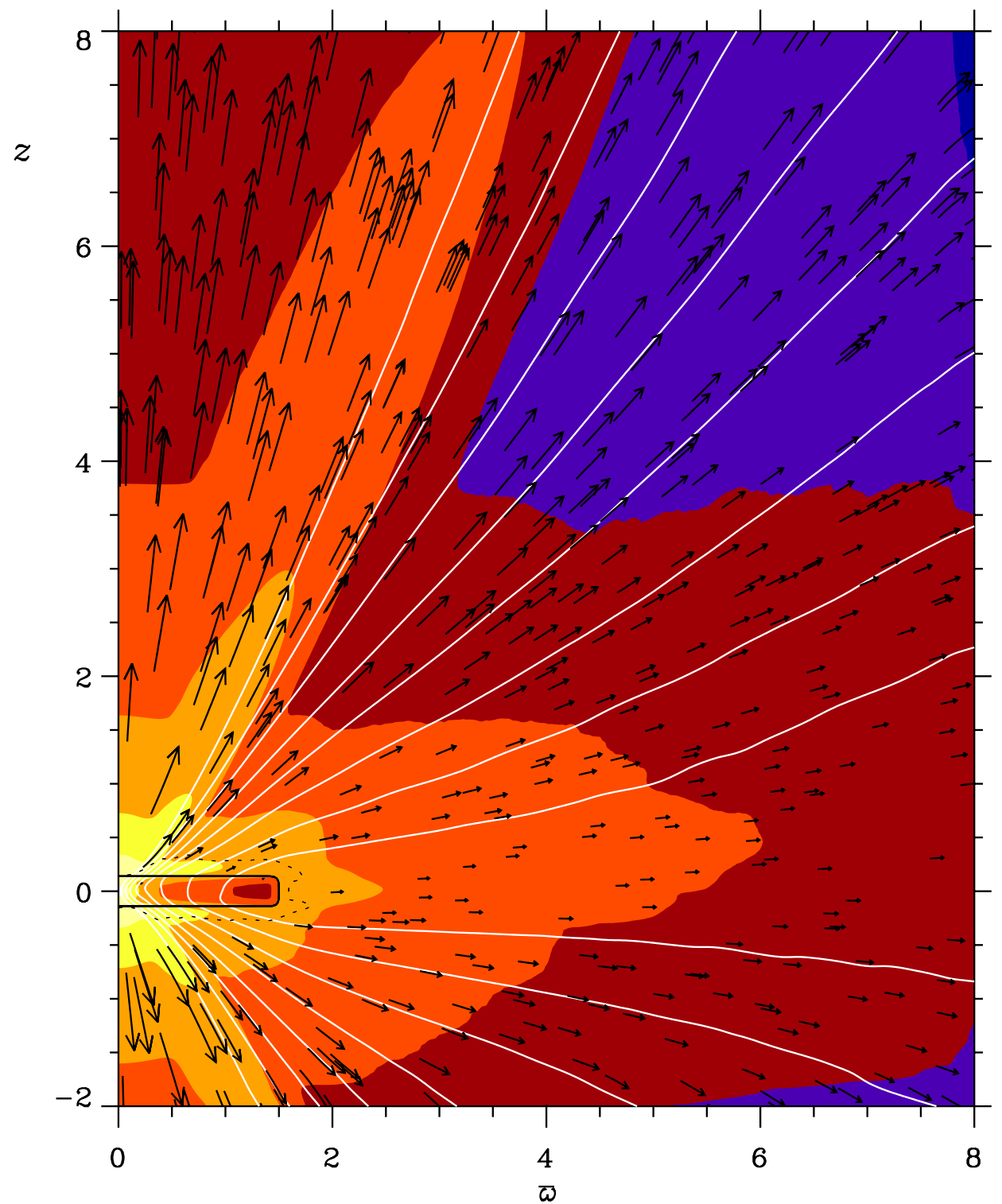

$\log h$

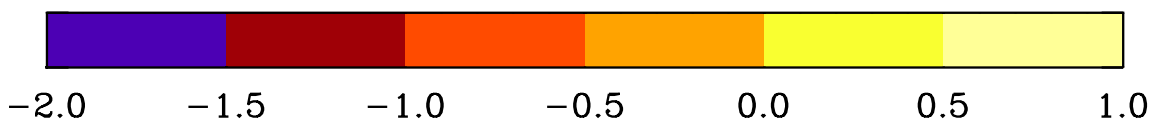

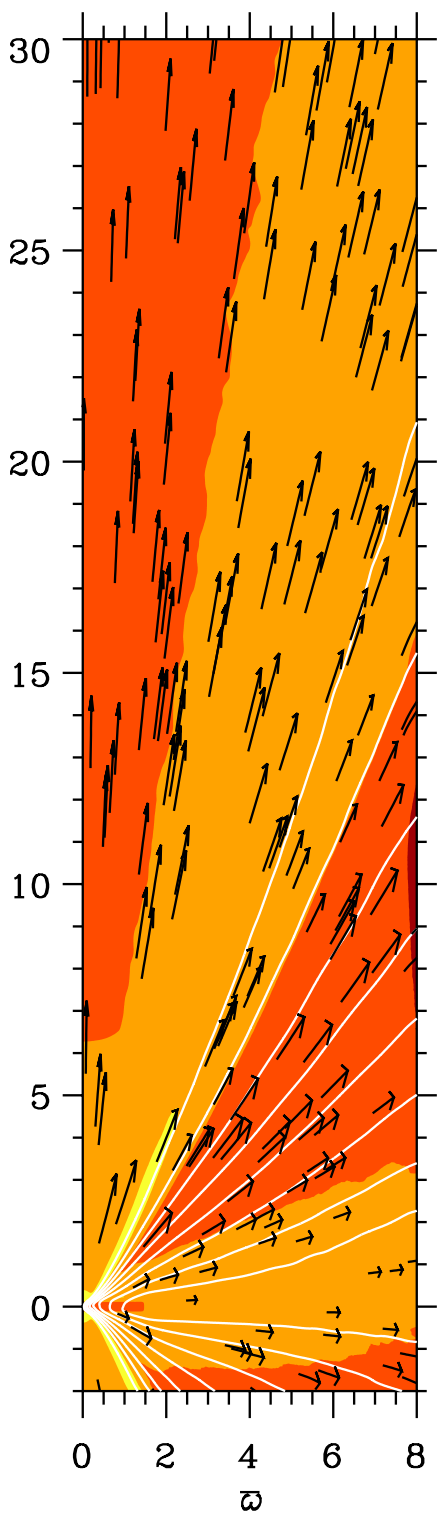

$\log h /|\Phi|$

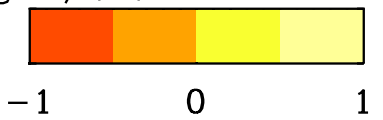

Fig. 6. Results for a larger domain, $[0,8] \times[-2,30]$, with the same parameters as in Fig. 4, averaged over times $t=200 \ldots 230, \beta=0.1$. Left panel: velocity vectors, poloidal magnetic field lines and grey scale representation of $h$ in the inner part of the domain. Right panel: velocity vectors, poloidal magnetic field lines and normalized specific enthalpy $h /|\Phi|$ in the full domain.

Our results are insensitive to the size and symmetry of the computational domain: we illustrate this in Fig. 6 with a larger domain of the size $[0,8] \times[-2,30]$. The disc midplane in this run is located asymmetrically in $z$ in order to verify that the (approximate) symmetry of the solutions is not imposed by the symmetry of the computational domain. Figure 6 confirms that our results are not affected by what happens near the computational boundaries.

Unlike the nonmagnetized system, the magnetized outflow changes only comparatively little when the mass sink is introduced at the centre. We show in Fig. 7 the results with a sink $\left(\tau_{\text {star }}=0.01\right)$ and otherwise the same parameters as in Fig. 4. As could be expected, the sink leads to a reduction in the outflow speed near the axis; the flow in the high speed cone becomes slower. But apart from that, the most important effects of the sink are the enhancement of the conical structure of the outflow and the smaller opening angle of the conical shell. A decrease in $\tau_{\text {star }}$ by a factor of 2 to 0.005 has very little effect, as illustrated in Figs. 8 and 10.

Increasing the entropy contrast (while keeping the specific entropy unchanged in the corona) reduces the opening angle of the conical shell. Pressure driving is obviously more important in this case, as compared to magneto-centrifugal driving (see Sect. 3.5). A model with $\beta=0.02$ (corresponding to a density and inverse temperature contrast of about 50:1 between the disc and the corona) is shown in Fig. 9. At $\vartheta=60^{\circ}$, the radial 


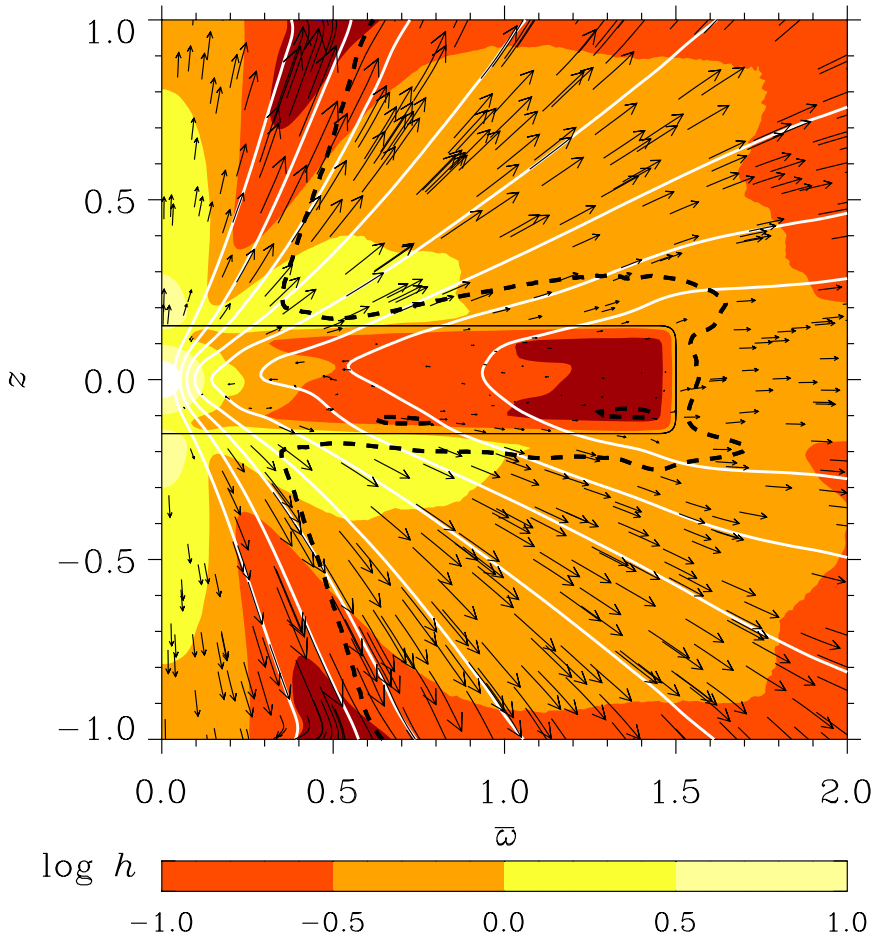

Fig. 7. As in Fig. 4, but with a mass sink at the centre, $\tau_{\text {star }}=0.01$. The outflow speed near the axis and the opening angle of the conical shell (now reaching $z= \pm 1$ at $\varpi \approx 0.5$ ) are reduced in comparison to that in the model without mass sink shown in Fig. 4, but the general structure of the outflow is little affected. Averaged over times $t=130 \ldots 140$, $\beta=0.1$. (Reference model.)

velocity $u_{\mathrm{r}}$ is slightly enhanced relative to the case $\beta=0.1$ (contrast 10:1); see Fig. 10. At $\vartheta=30^{\circ}$, on the other hand, the flow with the larger entropy contrast reaches the Alfvén point close to the disc (at $r \approx 0.27$ as opposed to $r \gtrsim 1$ in the other case), which leads to a smaller terminal velocity.

We conclude that the general structure of the magnetized flow and its typical parameters remain largely unaffected by the sink, provided its efficiency $\tau_{\text {star }}^{-1}$ does not exceed a certain threshold. It is plausibly the build-up of magnetic pressure at the centre that shields the central object to make the central accretion inefficient. This shielding would be even stronger if we included a magnetosphere of the central object. We discuss in Sect. 4.1 the dependence of our solution on the geometrical size of the sink and show that the general structure of the outflow persists as long as the size of the sink does not exceed the disk thickness.

\subsection{Magnetic field structure}

The dynamo in most of our models has $\alpha_{0}<0$, consistent with results from simulations of disc turbulence driven by the magneto-rotational instability (Brandenburg et al. 1995; Ziegler \& Rüdiger 2000). The resulting field symmetry is roughly dipolar, which seems to be typical of $\alpha \Omega$ disc dynamos with $\alpha_{0}<0$ in a conducting corona (e.g., Brandenburg et al. 1990). We note that the dominant parity of the magnetic field is sensitive to the magnetic diffusivity in the corona: a
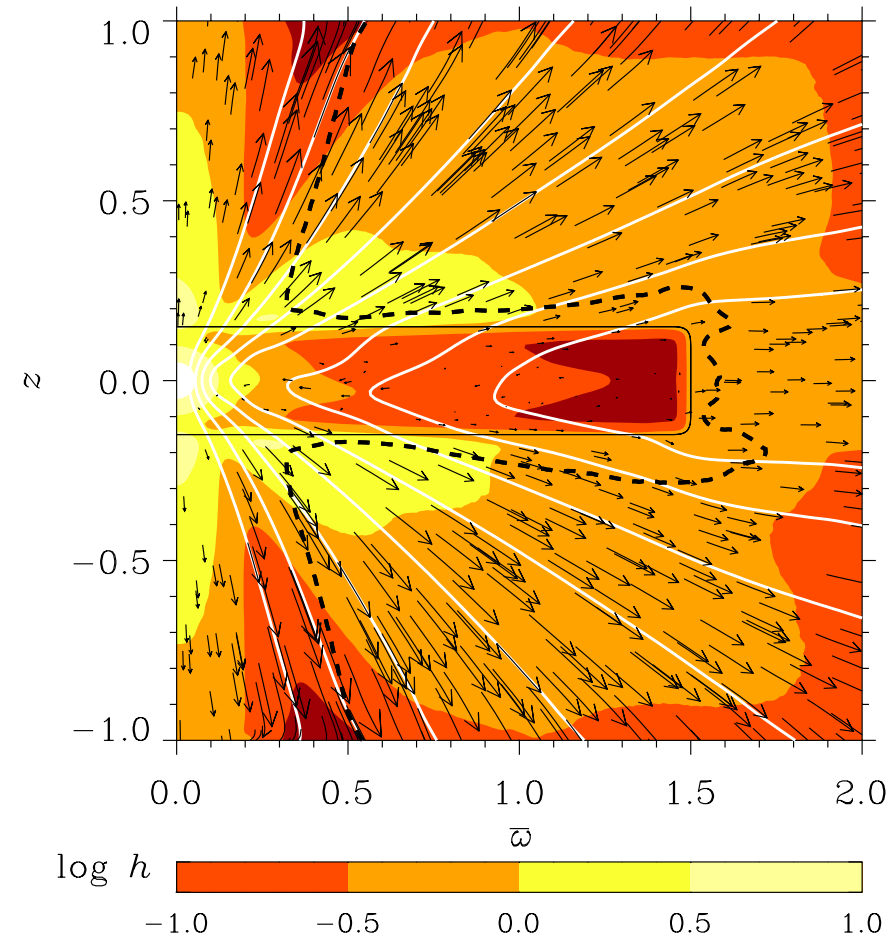

Fig. 8. As in Fig. 7, but with a stronger mass sink at the centre, $\tau_{\text {star }}=$ 0.005. The flow pattern is very similar to that of Fig. 7 where the time scale of the sink is twice as large. Averaged over times $t=122 \ldots 132$, $\beta=0.1$.

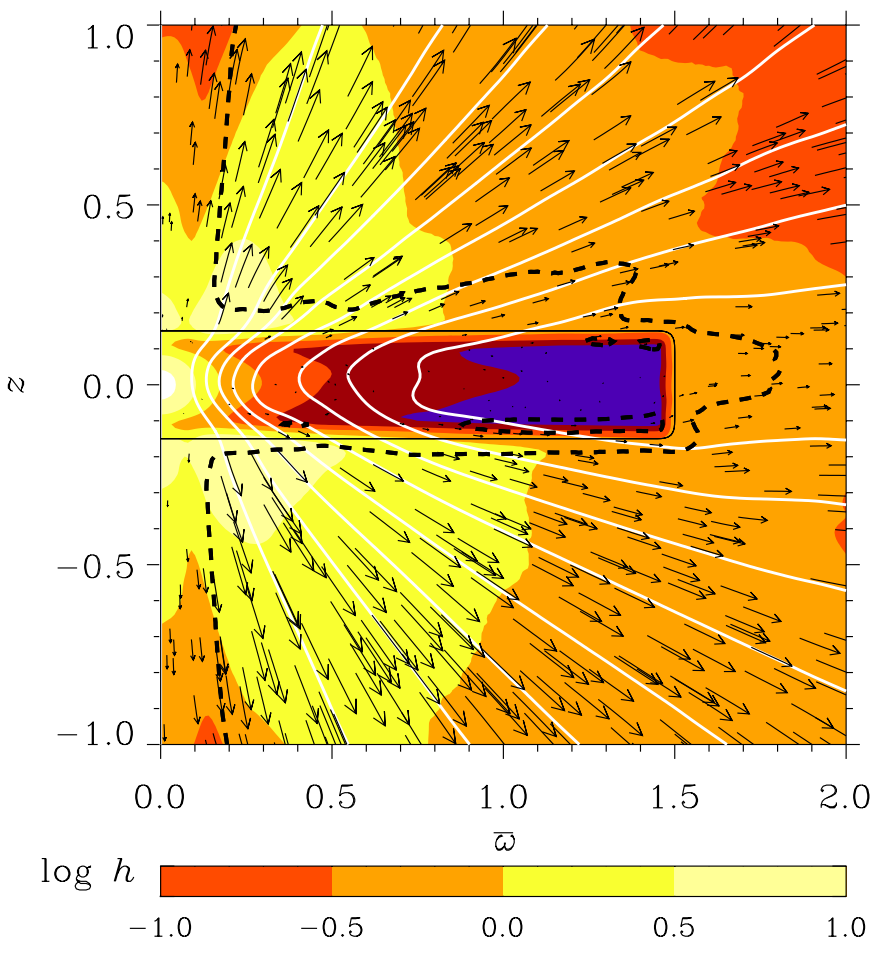

Fig. 9. As in Fig. 7, but with a larger entropy contrast, $\beta=0.02$. The opening angle of the conical shell is reduced in comparison to that of Figs. 7 and 8 where the entropy contrast is smaller (the shell crosses $z= \pm 1$ at $\varpi \approx 0.15$ ). Averaged over times $t=140 \ldots 240, \tau_{\text {star }}=0.01$.

quadrupolar oscillatory magnetic field dominates for $\alpha_{0}<0$ if the disc is surrounded by vacuum (Stepinski \& Levy 1988). 

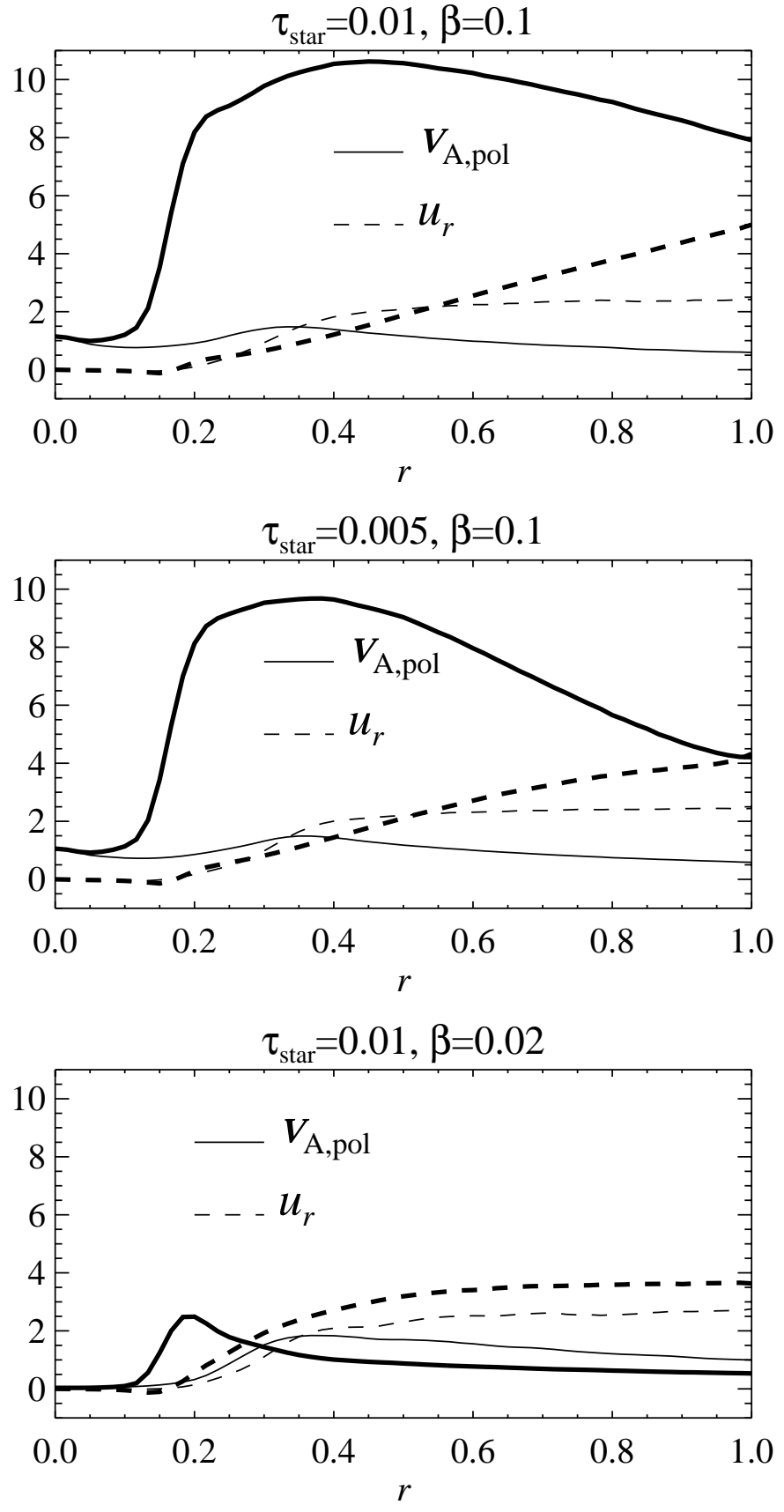

Fig. 10. Spherical radial velocity component, $u_{\mathrm{r}}$ (dashed), and poloidal Alfvén speed, $v_{\mathrm{A} \text {,pol }}$ (solid), as functions of spherical radius at polar angles $\vartheta=30^{\circ}$ (thick lines) and $\vartheta=60^{\circ}$ (thin lines) for the models of Figs. 7 (top panel), 8 (middle panel) and 9 (bottom panel).

For $\alpha_{0}<0$, the critical value of $\alpha_{0}$ for dynamo action is about 0.2 , which is a factor of about 50 larger than without outflows. Our dynamo is then only less than twice supercritical. A survey of the dynamo regimes for similar models is given by Bardou et al. (2001).

The initial magnetic field (poloidal, mixed parity) is weak $\left(p_{\text {mag }} \equiv \boldsymbol{B}^{2} /\left(2 \mu_{0}\right) \approx 10^{-5}\right)$, cf. Fig. 11 for comparison with the gas pressure], but the dynamo soon amplifies the field in the disc to $p_{\text {mag,tor }} \approx 10$, and then supplies it to the corona. As a result, the corona is filled with a predominantly azimuthal field with $p_{\text {mag,tor }} / p \approx 100$ at larger radii; see Fig. 11 . We note,
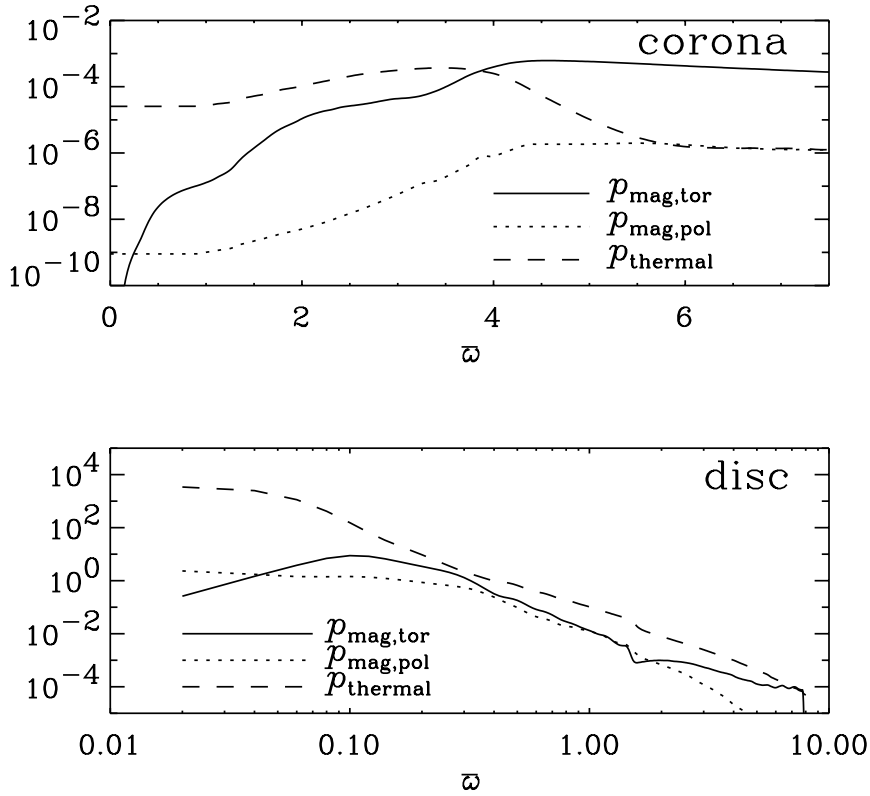

Fig. 11. Radial profiles of magnetic pressure from the toroidal (solid) and poloidal (dotted) magnetic fields and thermal pressure (dashed) for the model of Fig. 6 . Shown are the averages over the disc volume (lower panel) and over a region of the same size around $z=8$ in the corona (upper panel). $\beta=0.1$.

however, that the flow in the corona varies significantly in both space and time ${ }^{1}$. Magnetic pressure due to the toroidal field $B_{\varphi}$, $p_{\text {mag,tor }}$, exceeds gas pressure in the corona outside the inner cone and confines the outflow in the conical shell. The main mechanisms producing $B_{\varphi}$ in the corona are advection by the wind and magnetic buoyancy (cf. Moss et al. 1999). Magnetic diffusion and stretching of the poloidal field by vertical shear play a relatively unimportant rôle.

The field in the inner parts of the disc is dominated by the toroidal component; $\left|B_{\varphi}\right| B_{z} \mid \approx 3$ at $\varpi \lesssim 0.5$; this ratio is larger in the corona at all $\varpi$. However, as shown in Fig. 11, this ratio is closer to unity at larger radii in the disc.

As expected, $\alpha_{0}>0$ results in mostly quadrupolar fields (e.g., Ruzmaikin et al. 1988). As shown in Fig. 12, the magnetic field in the corona is now mainly restricted to a narrow conical shell that crosses $z= \pm 1$ at $\varpi \approx 0.6$. Comparing this figure with the results obtained with dipolar magnetic fields (Fig. 4), one sees that the quadrupolar field has a weaker effect on the outflow than the dipolar field; the conical shell is less pronounced. However, the structures within the inner cone are qualitatively similar to each other.

The magnitude and distribution of $\alpha$ in Eq. (14) only weakly affect magnetic field properties as far as the dynamo is saturated. For a saturated dynamo, the field distribution in the dynamo region $(0.2<\varpi<1.5,|z|<0.15)$ roughly follows from the equipartition field, $B \simeq\left(\varrho \mu_{0} v_{0}^{2}\right)^{1 / 2}$ with $v_{0}=c_{\mathrm{s}}$. In other words, nonlinear states of disc dynamos are almost insensitive to the detailed properties of $\alpha$ (e.g., Beck et al. 1996; Ruzmaikin et al. 1988).

\footnotetext{
1 See movie at http://www.nordita.dk/ brandenb/ movies/outflow
} 


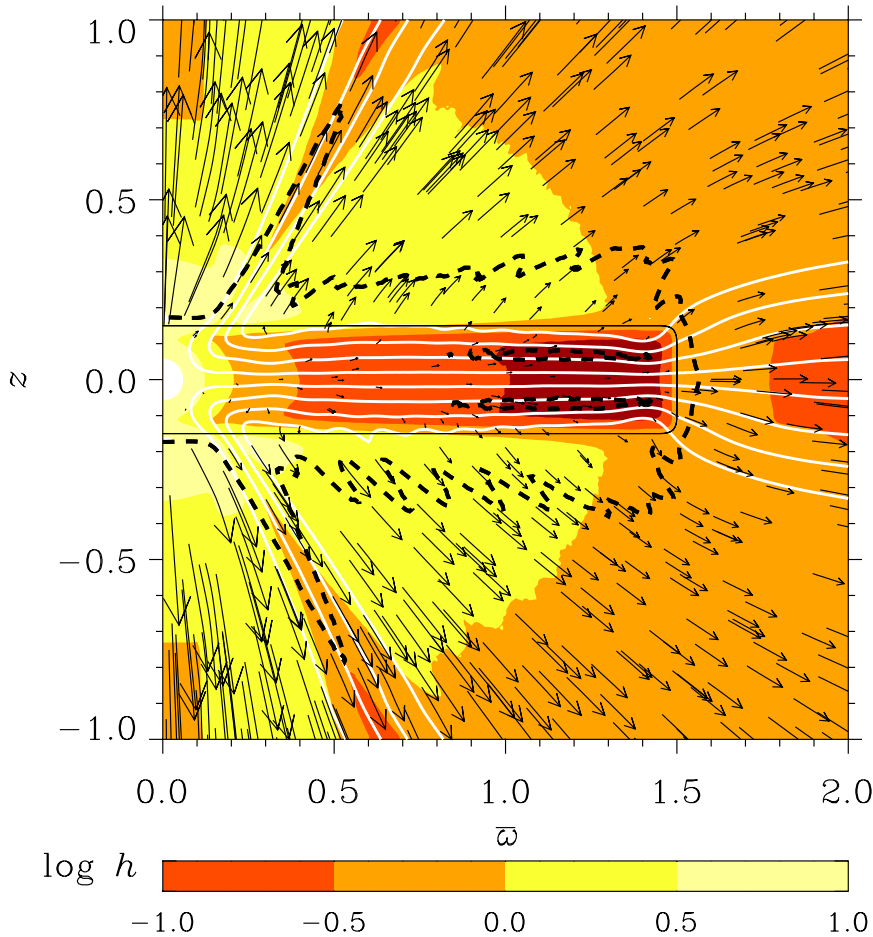

Fig. 12. As in Fig. 4, but with $\alpha_{0}=+0.3$, at time $t=132$. The magnetic field geometry is now mostly quadrupolar because $\alpha_{0}>0$.

A discussion of disc dynamos with outflows, motivated by the present model, can be found in Bardou et al. (2001). It is shown that the value of magnetic diffusivity in the corona does not affect the dynamo solutions strongly. Moreover, the outflow is fast enough to have the magnetic Reynolds number in the corona larger than unity, which implies that ideal integrals of motion are very nearly constant along field lines; see Sect. 3.6. The most important property is the sign of $\alpha$ as it controls the global symmetry of the magnetic field.

\subsection{Mass and energy loss}

The mass injection and loss rates due to the source, sink and wind are defined as

$\dot{M}_{\text {source }}=\int q_{\varrho}^{\text {disc }} \mathrm{d} V, \quad \dot{M}_{\text {sink }}=\int q_{\varrho}^{\text {star }} \mathrm{d} V$,

and

$\dot{M}_{\text {wind }}=\oint \varrho \boldsymbol{u} \cdot \mathrm{d} \boldsymbol{S}$,

respectively, where the integrals are taken over the full computational domain or its boundary. About $1 / 3$ of the mass released goes into the wind and the rest is accreted by the sink, in the model with $\tau_{\text {star }}=0.01$ and $\beta=0.1$ of Fig. 7 . Reducing $\tau_{\text {star }}$ by a factor of 2 (as in the model of Fig. 8), only changes the global accretion parameters by a negligible amount $(\lesssim 10 \%)$.

The mass loss rate in the wind fluctuates on a time scale of 5 time units, but remains constant on average at about $\dot{M}_{\text {wind }} \approx$ 3 , corresponding to $6 \times 10^{-7} M_{\odot} \mathrm{yr}^{-1}$, in the models of Figs. 7 and 8 . The mass in the disc, $M_{\text {disc }}$, remains roughly constant.
The rate at which mass needs to be replenished in the disc, $\dot{M}_{\text {source }} / M_{\text {disc }}$, is about 0.4 . This rate is not controlled by the imposed response rate of the mass source, $\tau_{\text {disc }}^{-1}$, which is 25 times larger. So, the mass source adjusts itself to the disc evolution and does not directly control the outflow. We show in Fig. 13 trajectories that start in and around the mass injection region. The spatial distribution of the mass replenishment rate $q_{\varrho}^{\text {disc }}$ shown in Fig. 13 indicates that the mass is mainly injected close to the mass sink, and $q_{\varrho}^{\text {disc }}$ remains moderate in the outer parts of the disc. (Note that the reduced effect of the mass sink in the magnetized flow is due to magnetic shielding rather than to mass replenishment near the sink - see Sect. 3.2.)

The angular structure of the outflow can be characterized by the following quantities calculated for a particular spherical radius, $r=8$, for the model of Fig. 6: the azimuthally integrated normalized radial mass flux density, $\dot{M}(\vartheta) / M_{\text {disc }}$, where

$\dot{M}(\vartheta)=2 \pi r^{2} \varrho u_{\mathrm{r}} \sin \vartheta, \quad M_{\mathrm{disc}}=\int_{\mathrm{disc}} \rho \mathrm{d} V$

the azimuthally integrated normalized radial angular momentum flux density, $\dot{J}(\vartheta) / J_{\text {disc }}$, where

$\dot{J}(\vartheta)=2 \pi r^{2} \varrho \varpi u_{\varphi} u_{\mathrm{r}} \sin \vartheta, \quad J_{\text {disc }}=\int_{\text {disc }} \rho \varpi u_{\varphi} \mathrm{d} V$

the azimuthally integrated normalized radial magnetic energy flux (Poynting flux) density, $\dot{E}_{\mathrm{M}}(\vartheta) / E_{\mathrm{M}}$, where

$\dot{E}_{\mathrm{M}}(\vartheta)=2 \pi r^{2} \frac{(\boldsymbol{E} \times \boldsymbol{B})_{\mathrm{r}}}{\mu_{0}} \sin \vartheta, \quad E_{\mathrm{M}}=\int_{\mathrm{disc}} \frac{\boldsymbol{B}^{2}}{2 \mu_{0}} \mathrm{~d} V$,

and the azimuthally integrated normalized radial kinetic energy flux density, $\dot{E}_{\mathrm{K}}(\vartheta) / E_{\mathrm{K}}$, where

$\dot{E}_{\mathrm{K}}(\vartheta)=2 \pi r^{2}\left(\frac{1}{2} \varrho \boldsymbol{u}^{2} u_{\mathrm{r}}\right) \sin \vartheta, \quad E_{\mathrm{K}}=\int_{\mathrm{disc}} \frac{1}{2} \rho \boldsymbol{u}^{2} \mathrm{~d} V$.

Here, $M_{\text {disc }}, J_{\text {disc }}, E_{\mathrm{M}}$ and $E_{\mathrm{K}}$ are the mass, angular momentum, and magnetic and kinetic energies in the disc. A polar diagram showing these distributions is presented in Fig. 14 for the model of Fig. 6. Note that this is a model without central mass sink where the flow in the hot, dense cone around the axis is fast. The fast flow in the hot, dense cone carries most of the mass and kinetic energy. A significant part of angular momentum is carried away in the disc plane whilst the magnetic field is ejected at intermediate angles, where the conical shell is located.

The radial kinetic and magnetic energy flux densities, integrated over the whole sphere, are $L_{\mathrm{K}} \equiv \int_{0}^{\pi} \dot{E}_{\mathrm{K}}(\vartheta) \mathrm{d} \vartheta \approx$ $0.54 \dot{M}_{\text {wind }} c_{*}^{2}$ and $L_{\mathrm{M}} \equiv \int_{0}^{\pi} \dot{E}_{\mathrm{M}}(\vartheta) \mathrm{d} \vartheta \approx 0.03 \dot{M}_{\text {wind }} c_{*}^{2}$, respectively, where $c_{*} \approx 2.8$ is the fast magnetosonic speed (with respect to the poloidal field) at the critical surface (where $u_{z}=c_{*}$ ) on the axis. Thus, $\dot{M}_{\text {wind }} c_{*}^{2}$ can be taken as a good indicator of the kinetic energy loss, and the magnetic energy loss into the exterior is about $3 \%$ of this value. These surface-integrated flux densities (or luminosities) are, as expected, roughly independent of the distance from the central object. 


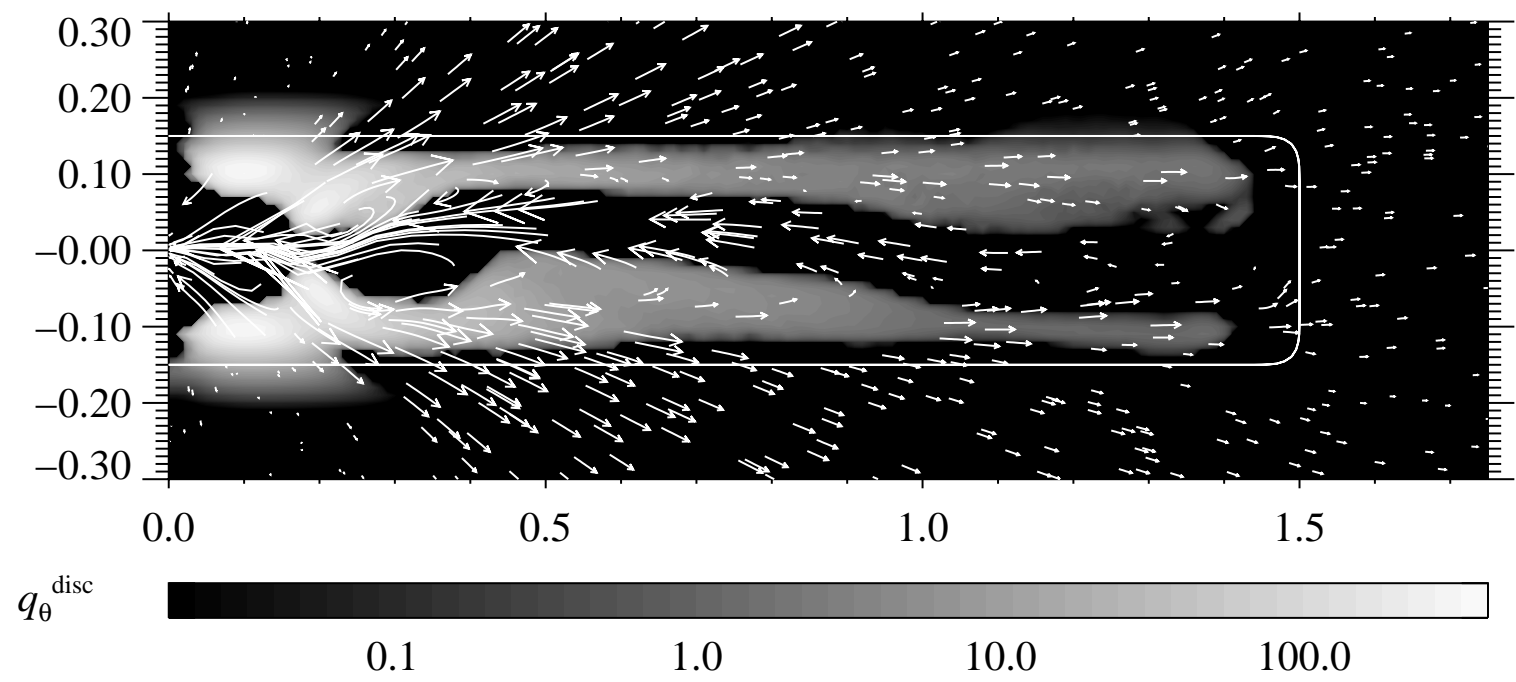

Fig. 13. Azimuthally integrated mass flux density, represented as a vector $2 \pi \varpi \varrho\left(u_{\varpi}, u_{z}\right)$, in the simulation of Fig. 7 with a dipolar magnetic field and a mass sink at the centre. Shades of grey show the distribution of the mass source in the disc, $q_{\varrho}^{\text {disc }}$. The disc boundary is shown with a white line.

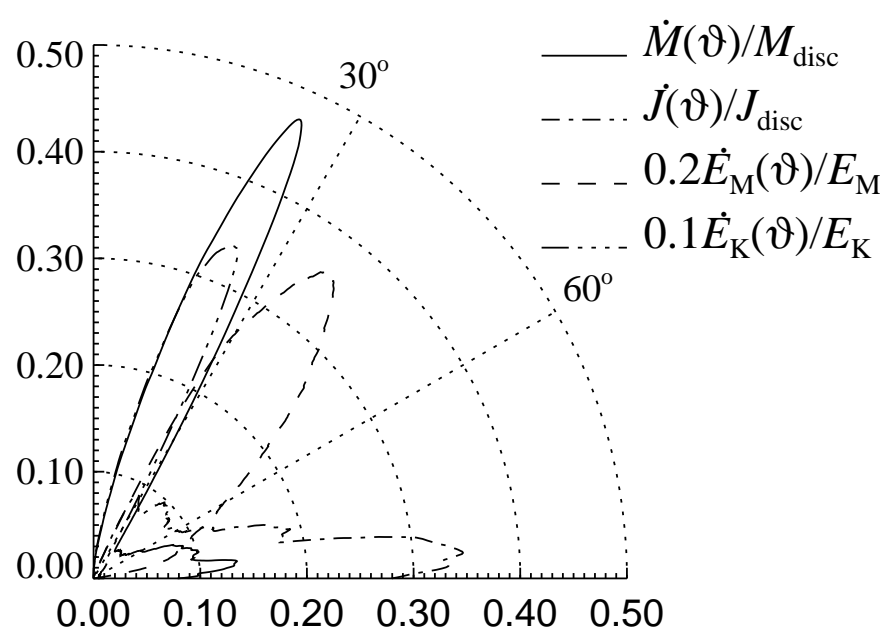

Fig. 14. Dependence, on polar angle $\vartheta$, of azimuthally integrated radial mass flux density $\dot{M}(\vartheta)$ through a sphere $r=8$ (solid, normalized by the disc mass $M_{\text {disc }} \approx 12$ ), azimuthally integrated radial angular momentum flux density $\dot{J}(\vartheta)$ (dashed-dotted, normalized by the disc angular momentum $J_{\text {disc }} \approx 4.3$ ), azimuthally integrated radial Poynting flux density $\dot{E}_{\mathrm{M}}(\vartheta)$ (divided by 5 , dashed, normalized by the magnetic energy in the disc $E_{\mathrm{M}} \approx 0.6$ ), and azimuthally integrated radial kinetic energy flux density $\dot{E}_{\mathrm{K}}(\vartheta)$ (divided by 10 , dash-3dots, normalized by the kinetic energy in the disc $E_{\mathrm{K}} \approx 9.7$ ), for the model of Fig. 6 . The unit of all the quantities is $[t]^{-1} \mathrm{rad}^{-1}$.

\subsection{Mechanisms of wind acceleration}

The magnetized outflows in our models with central mass sink have a well-pronounced structure, with a fast, cool and lowdensity flow in a conical shell, and a slower, hotter and denser flow near the axis and in the outer parts of the domain. Without central mass sink, there is a high speed, hot and dense cone around the axis.

The magnetic field geometry (e.g., Fig. 7) is such that for $\varpi>0.1$, the angle between the disc surface and the field lines is less than $60^{\circ}$, reaching $\approx 20^{\circ}$ at $\varpi \approx 1-1.5$, which is

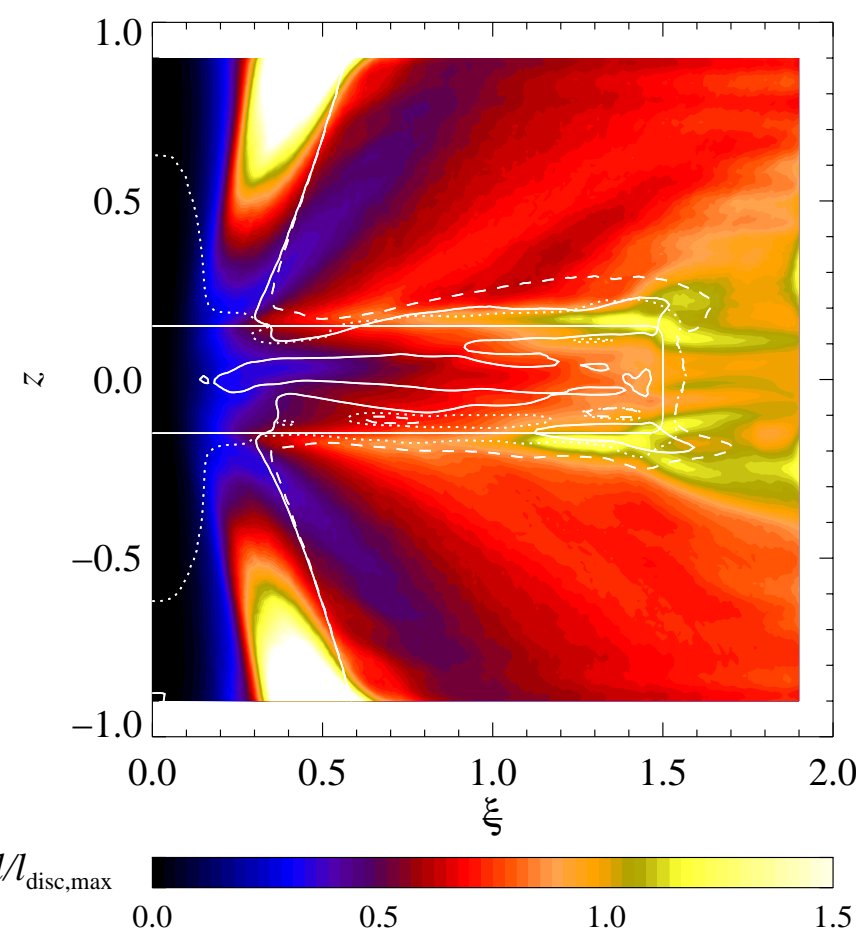

Fig. 15. Angular momentum (normalized by the maximum angular momentum in the disc) for the model with $\tau_{\text {star }}=0.01$ and $\beta=0.1$, shown in Fig. 7 . The maximum value is $l / l_{\mathrm{disc, \text {max }}} \approx 2.5$. The dashed line shows the fast magnetosonic surface with respect to the poloidal field (cf. Fig. 4), the solid line the Alfvén surface where the poloidal velocity equals the poloidal Alfvén speed, and the dotted line the sonic surface. Averaged over times $t=130 \ldots 140$.

favourable for magneto-centrifugal acceleration (Blandford \& Payne 1982; Campbell 1999, 2000, 2001). However, the Alfvén surface is so close to the disc surface in the outer parts of the disc that acceleration there is mainly due to pressure gradient. The situation is, however, different in the conical shell containing the fast wind. As can be seen from Fig. 15, the Alfvén 


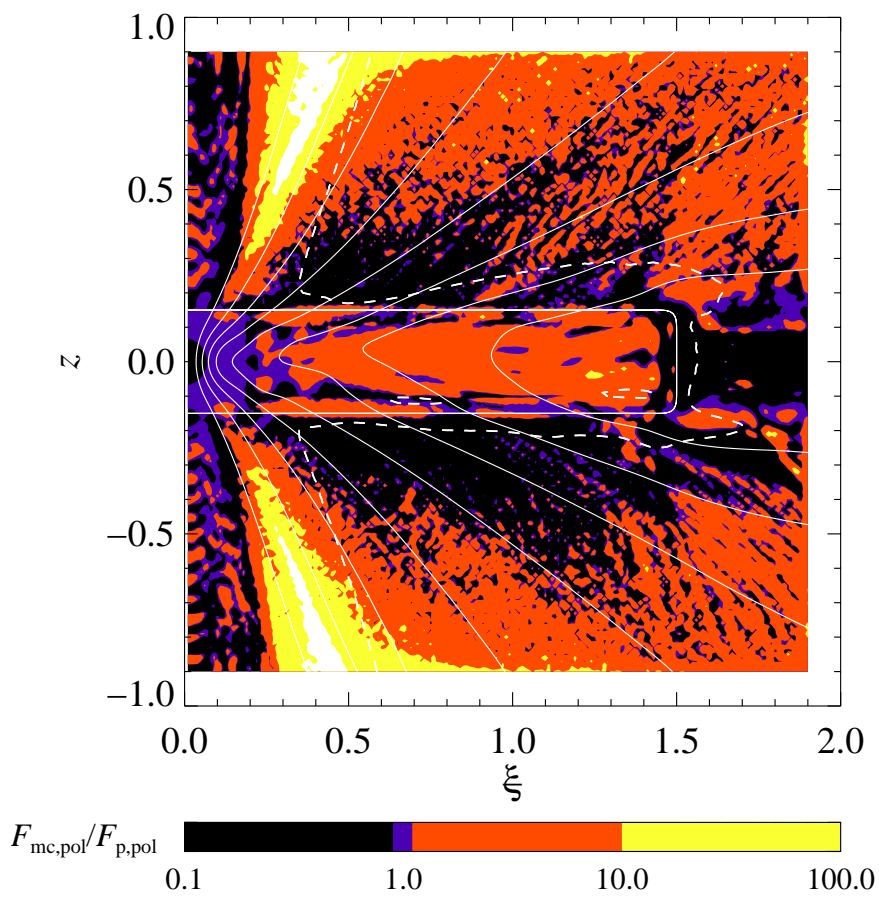

Fig. 16. The ratio of the poloidal magneto-centrifugal and pressure forces, $\left|\boldsymbol{F}_{\mathrm{pol}}^{(\mathrm{mc})}\right| /\left|\boldsymbol{F}_{\mathrm{pol}}^{(\mathrm{p})}\right|$ as defined in Eq. (42), is shown with shades of grey, with larger values corresponding to lighter shades. The maximum value is $\left|\boldsymbol{F}_{\mathrm{pol}}^{(\mathrm{mc})}\right| /\left|\boldsymbol{F}_{\mathrm{pol}}^{(\mathrm{p})}\right| \approx 411$. Superimposed are the poloidal magnetic field lines. The dashed line shows the fast magnetosonic surface with respect to the poloidal field (cf. Fig. 4). The parameters are as in the model of Fig. 7. Averaged over times $t=130 \ldots 140$.

surface is far away from the disc in that region and, on a given field line, the Alfvén radius is at least a few times larger than the radius of the footpoint in the disc. This is also seen in simulations of the magneto-centrifugally driven jets of Krasnopolsky et al. (1999); see their Fig. 4. The lever arm of about 3 is sufficient for magneto-centrifugal driving to dominate. As can be seen also from Fig. 10, the flow at the polar angle $\vartheta=60^{\circ}$ is mainly accelerated by pressure gradient near the disc surface (where the Alfvén surface is close to the disc surface). However, acceleration remains efficient out to at least $r=1$ within the conical shell at $\vartheta \approx 30^{\circ}$. This can be seen in the upper and middle panels of Fig. 10 (note that the conical shell is thinner and at a smaller $\vartheta$ in the model with larger entropy contrast, and so it cannot be seen in this figure, cf. Fig. 9). These facts strongly indicate that magneto-centrifugal acceleration dominates within the conical shell.

Another indicator of magneto-centrifugal acceleration in the conical shell is the distribution of angular momentum (see Fig. 15), which is significantly larger in the outer parts of the conical shell than in the disc, which suggests that the magnetic field plays an important rôle in the flow dynamics. We show in Fig. 16 the ratio of the "magneto-centrifugal force" to pressure gradient, $\left|\boldsymbol{F}_{\text {pol }}^{(\mathrm{mc})}\right| /\left|\boldsymbol{F}_{\text {pol }}^{(\mathrm{p})}\right|$, where the subscript "pol" denotes the poloidal components. Here, the "magneto-centrifugal force" includes all terms in the poloidal equation of motion, except for the pressure gradient (but we ignore the viscous term

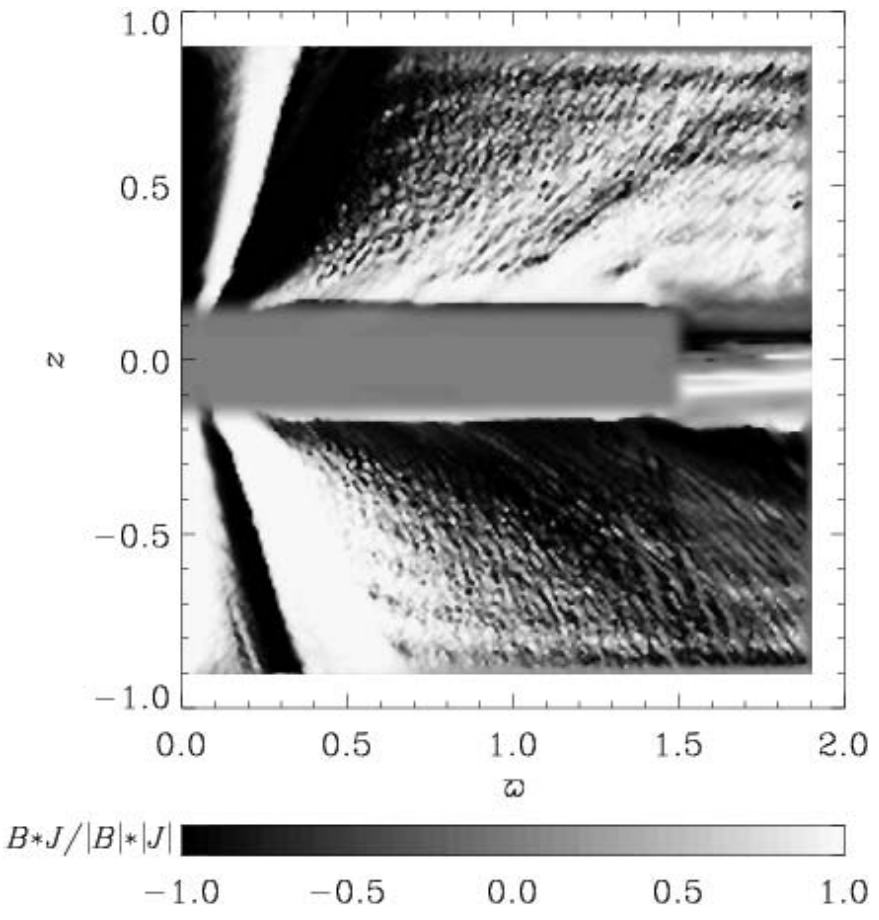

Fig. 17. The ratio $\boldsymbol{J} \cdot \boldsymbol{B} /|\boldsymbol{J}||\boldsymbol{B}|$ in the corona for the model with $\tau_{\text {star }}=$ 0.01 and $\beta=0.1$, shown in Fig. 7. For a force-free magnetic field, $\boldsymbol{J}=\boldsymbol{C} \boldsymbol{B}$, this ratio is \pm 1 . Averaged over times $t=130 \ldots 140$.

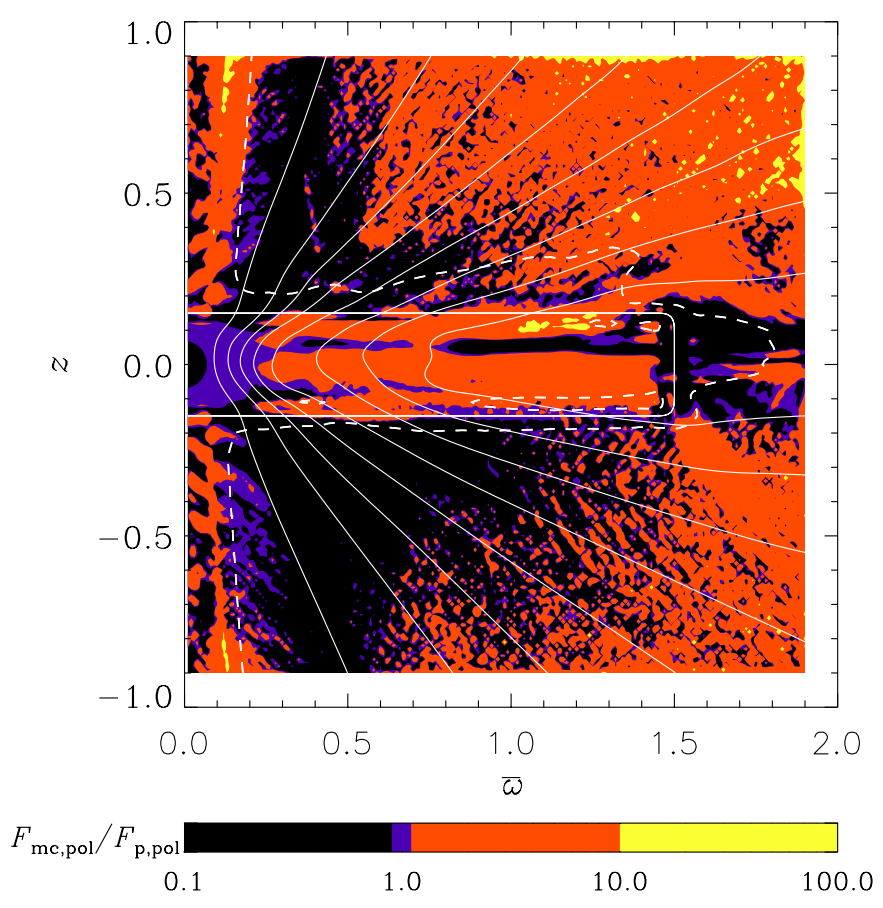

Fig. 18. As in Fig. 16, but for the model of Fig. 9, i.e. with larger entropy contrast, $\beta=0.02$. Averaged over times $t=140 \ldots 240$.

and the mass production term, the latter being restricted to the disc only),

$\boldsymbol{F}^{(\mathrm{mc})}=\varrho\left(\Omega^{2} \varpi-\nabla \Phi\right)+\boldsymbol{J} \times \boldsymbol{B}$, 
and $\boldsymbol{F}^{(p)}=-\boldsymbol{\nabla} p$. The large values of the ratio in the conical shell confirm that magneto-centrifugal driving is dominant there. On the other hand, the pressure gradient is strong enough in the outer parts of the disc to shift the Alfvén surface close to the disc surface, leading to pressure driving. This is also discussed in Casse \& Ferreira (2000b) who point out that, although the criterion of Blandford \& Payne (1982) is fulfilled, thermal effects can be strong enough to lead to pressure driving. According to Ferreira (1997), a decrease of the total poloidal current $I_{\mathrm{pol}}=2 \pi \varpi B_{\varphi} / \mu_{0}$ along a field line is another indicator of magneto-centrifugal acceleration. We have compared the poloidal current $I_{\mathrm{pol}}^{\text {(top) }}$ at the Alfvén point, or at the top of our box if the Alfvén point is outside the box, with the poloidal current $I_{\text {pol }}^{\text {(sur) }}$ at the disc surface, and find that outside the conical shell this ratio is typically $\geq 0.8$, while along the field line that leaves the box at $(\varpi, z)=(0.6,1)$, we get $I_{\text {pol }}^{\text {(top) }} / I_{\text {pol }}^{\text {(surf }} \approx 0.18$, i.e. a strong reduction, which confirms that magneto-centrifugal acceleration occurs inside the conical shell. We note, however, that the changing sign of $B_{\varphi}$ and therefore of $I_{\mathrm{pol}}$ makes this analysis inapplicable in places, and the distribution of angular momentum (Fig. 15) gives a much clearer picture.

As further evidence of a significant contribution from magneto-centrifugal driving in the conical shell, we show in Fig. 17 that the magnetic field is close to a force-free configuration in regions where angular momentum is enhanced, i.e. in the conical shell and in the corona surrounding the outer parts of the disc. These are the regions where the Lorentz force contributes significantly to the flow dynamics, so that the magnetic field performs work and therefore relaxes to a force-free configuration. The radial variation in the sign of the current helicity $\boldsymbol{J} \cdot \boldsymbol{B}$ is due to a variation in the sign of the azimuthal magnetic field and of the current density. Such changes originate from the disc where they imprint a corresponding variation in the sign of the angular momentum constant, see Eq. (46). These variations are then carried along magnetic lines into the corona. The locations where the azimuthal field reverses are still relatively close to the axis, and there the azimuthal field relative to the poloidal field is weak compared to regions further away from the axis.

Pressure driving is more important if the entropy contrast between the disc and the corona is larger (i.e. $\beta$ is smaller): the white conical shell in Fig. 16 indicative of stronger magnetocentrifugal driving shifts to larger heights for $\beta=0.02$, as shown in Fig. 18. We note, however, that there are periods when magneto-centrifugal driving is dominant even in this model with higher entropy contrast, but pressure driving dominates in the time averaged picture (at least within our computational domain).

We show in Fig. 19 the variation of several quantities along a magnetic field line that has its footpoint at the disc surface at $(\varpi, z)=(0.17,0.15)$ and lies around the conical shell. Since this is where magneto-centrifugal driving is still dominant, it is useful to compare Fig. 19 with Fig. 3 of Ouyed et al. (1997), where a well-collimated magneto-centrifugal jet is studied. Since our outflow is collimated only weakly within our computational domain, the quantities are plotted against height $z$, rather than $z / \varpi$ as in Ouyed et al. (1997) $(z / \varpi$ is
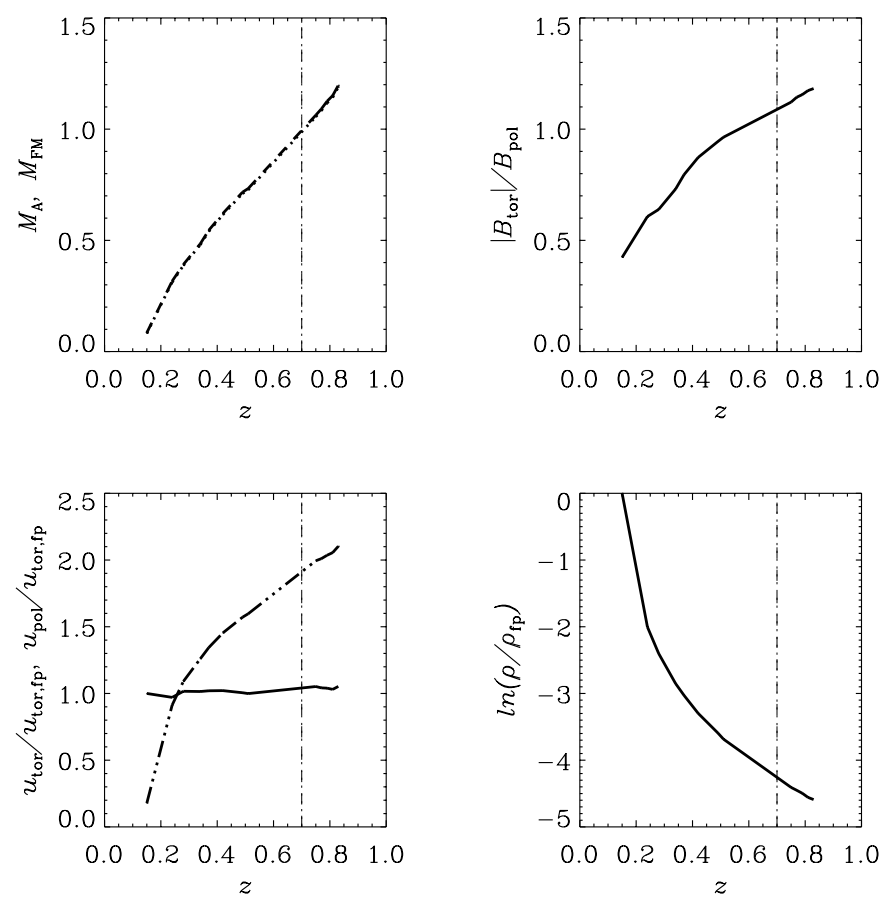

Fig. 19. Flow parameters as functions of height $z$, along the field line with its footpoint at $\left(\varpi_{\mathrm{fp}}, z_{\mathrm{fp}}\right)=(0.17,0.15)$, for the model of Fig. 7 . Upper left: poloidal Alfvén Mach number, $M_{\mathrm{A}} \equiv u_{\mathrm{pol}} / v_{\mathrm{A} \text {,pol }}$ (dashed), and a similar quantity that includes the poloidal Alfvén speed as well as the sound speed (fast magnetosonic Mach number with respect to the poloidal field), $M_{\mathrm{FM}} \equiv u_{\mathrm{pol}} /\left(c_{\mathrm{s}}^{2}+v_{\mathrm{Apol}}^{2}\right)^{1 / 2}$ (dotted) - the two curves are practically identical; upper right: ratio of toroidal $\left(B_{\text {tor }}\right)$ and poloidal $\left(B_{\text {pol }}\right)$ magnetic fields; lower left: toroidal velocity $u_{\text {tor }}$ (solid) and poloidal velocity $u_{\mathrm{pol}}$ (dash-3dots), in units of the toroidal velocity at the footpoint, $u_{\mathrm{tor}, \mathrm{fp}}$; lower right: density $\varrho$ in units of the density at the footpoint, $\varrho_{\mathrm{fp}}$. The position of the Alfvén (and fast magnetosonic) point on the field line is indicated by the vertical line. This figure is useful to compare with Fig. 3 of Ouyed et al. (1997).

nearly constant along a field line for weakly collimated flows, whereas approximately $z / \varpi \propto z$ along a magnetic line for wellcollimated flows). The results are qualitatively similar, with the main difference that the fast magnetosonic surface in our model almost coincides with the Alfvén surface in the region around the conical shell where the outflow is highly supersonic. Since we include finite diffusivity, the curves in Fig. 19 are smoother than in Ouyed et al. (1997), who consider ideal MHD. A peculiar feature of the conical shell is that the flow at $z \lesssim 1$ is sub-Alfvénic but strongly supersonic. The fast magnetosonic surface is where the poloidal velocity $v_{\text {pol }}$ equals the fast magnetosonic speed for the direction parallel to the field lines,

$v_{\mathrm{pol}}^{2}=\frac{1}{2}\left(c_{\mathrm{s}}^{2}+v_{\mathrm{A}}^{2}+\sqrt{\left(c_{\mathrm{s}}^{2}+v_{\mathrm{A}}^{2}\right)^{2}-4 c_{\mathrm{s}}^{2} v_{\mathrm{A}, \mathrm{pol}}^{2}}\right)$,

with $v_{\mathrm{A}, \mathrm{pol}}$ the Alfvén speed from the poloidal magnetic field. This surface has the same overall shape as the fast magnetosonic surface with respect to the poloidal field, albeit in some cases it has a somewhat larger opening angle around the conical shell and is located further away from the disc in regions where the toroidal Alfvén speed is enhanced. 


\subsection{Lagrangian invariants}

Axisymmetric ideal magnetized outflows are governed by five Lagrangian invariants, the flux ratio,

$k=\varrho u_{z} / B_{z}=\varrho u_{\varpi} / B_{\varpi}$,

the angular velocity of magnetic field lines,

$\widetilde{\Omega}=\varpi^{-1}\left(u_{\varphi}-k B_{\varphi} / \varrho\right)$,

the angular momentum constant,

$\ell=\varpi u_{\varphi}-\varpi B_{\varphi} /\left(\mu_{0} k\right)$,

the Bernoulli constant,

$e=\frac{1}{2} \boldsymbol{u}^{2}+h+\Phi-\varpi \widetilde{\Omega} B_{\varphi} /\left(\mu_{0} k\right)$

and specific entropy $s$ (which is a prescribed function of position in our model). In the steady state, these five quantities are conserved along field lines, but vary from one magnetic field line to another (e.g., Pelletier \& Pudritz 1992; Mestel 1999), i.e. depend on the magnetic flux within a magnetic flux surface, $2 \pi a$, where $a=\varpi A_{\varphi}$ is the flux function whose contours represent poloidal field lines.

We show in Fig. 20 scatter plots of $k(a), \widetilde{\Omega}(a), \ell(a)$, and $e(a)$ for the model of Fig. 6. Points from the region $0.2 \leq z \lesssim 8$ collapse into a single line, confirming that the flow in the corona is nearly ideal ${ }^{2}$. This is not surprising since the magnetic Reynolds number is much greater than unity in the corona for the parameters adopted here. For $8 \lesssim z \leq 30$, there are departures from perfect MHD; in particular, the angular velocity of magnetic field lines, $\widetilde{\Omega}$, is somewhat decreased in the upper parts of the domain (indicated by the vertical scatter in the data points). This is plausibly due to the finite magnetic diffusivity which still allows matter to slightly lag behind the magnetic field. As this lag accumulates along a stream line, the departures increase with height $z$. Since this is a "secular" effect only, and accumulates with height, we locally still have little variation of $k$ and $\widetilde{\Omega}$, which explains why magneto-centrifugal acceleration can operate quite efficiently.

The corona in our model has (turbulent) magnetic diffusivity comparable to that in the disc. This is consistent with, e.g., Ouyed \& Pudritz (1999) who argue that turbulence should be significant in coronae of accretion discs. Nevertheless, it turns out that ideal MHD is a reasonable approximation for the corona (see Fig. 20), but not for the disc. Therefore, magnetic diffusivity is physically significant in the disc and insignificant in the corona (due to different velocity and length scales involved), as in most models of disc outflows (see Ferreira \& Pelletier 1995 for a discussion). Thus, our model confirms this widely adopted idealization.

${ }^{2}$ If we restrict ourselves to $0.2 \leq z \lesssim 8$, about $90 \%$ of the points for $\widetilde{\Omega}(a)$ are within $\pm 10 \%$ of the line representing $z=4$; for the other three invariants, this percentage is at least $97 \%$. In a larger domain, $0.2 \leq z \lessgtr 30$, these percentages drop to about $80 \%$ for $k(a)$ and $60 \%$ for $\widetilde{\Omega}(a)$; for $\ell(a)$ and $e(a)$, however, they remain greater than $90 \%$.
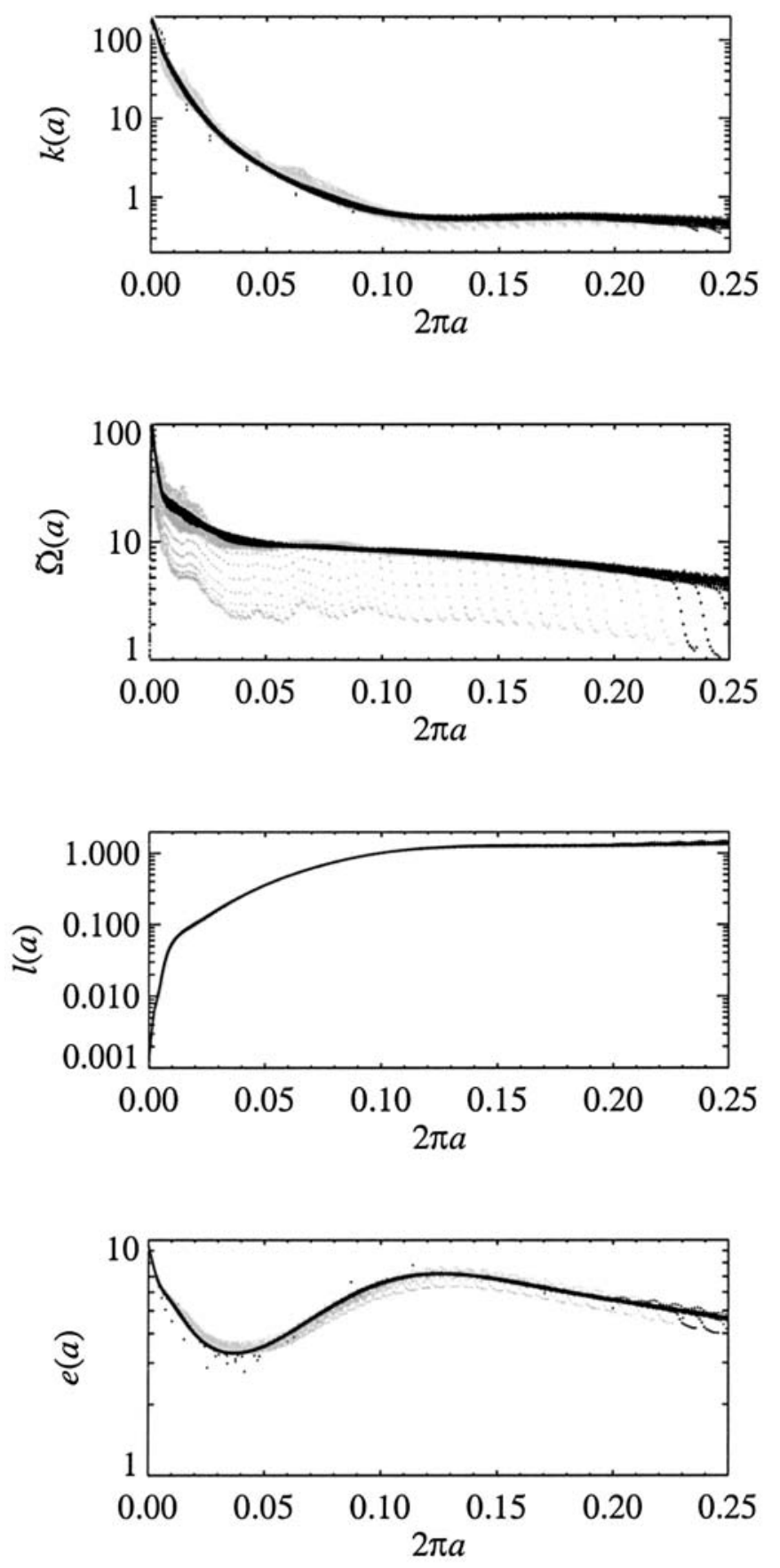

Fig. 20. The four Lagrangian invariants $k(a), \widetilde{\Omega}(a), \ell(a)$, and $e(a)$ as a function of the flux function $a$ for the model of Fig. 6 . All points in the domain $0<\varpi<8,0.2 \leq z \leq 30$ are shown (provided $0<a \leq$ 0.25 ). The dots deviating from well-defined curves mostly originate in $8 \lesssim z \leq 30$. Note that specific entropy as fifth Lagrangian invariant is trivially conserved in our model, because entropy is spatially constant throughout the corona and temporally fixed.

\section{Toward more realistic models}

The models presented so far have some clear deficiencies when compared with characteristic features relevant to protostellar discs. The first deficiency concerns the relative amount of matter accreted onto the central object compared with what goes into the wind. A typical figure from the models presented above 


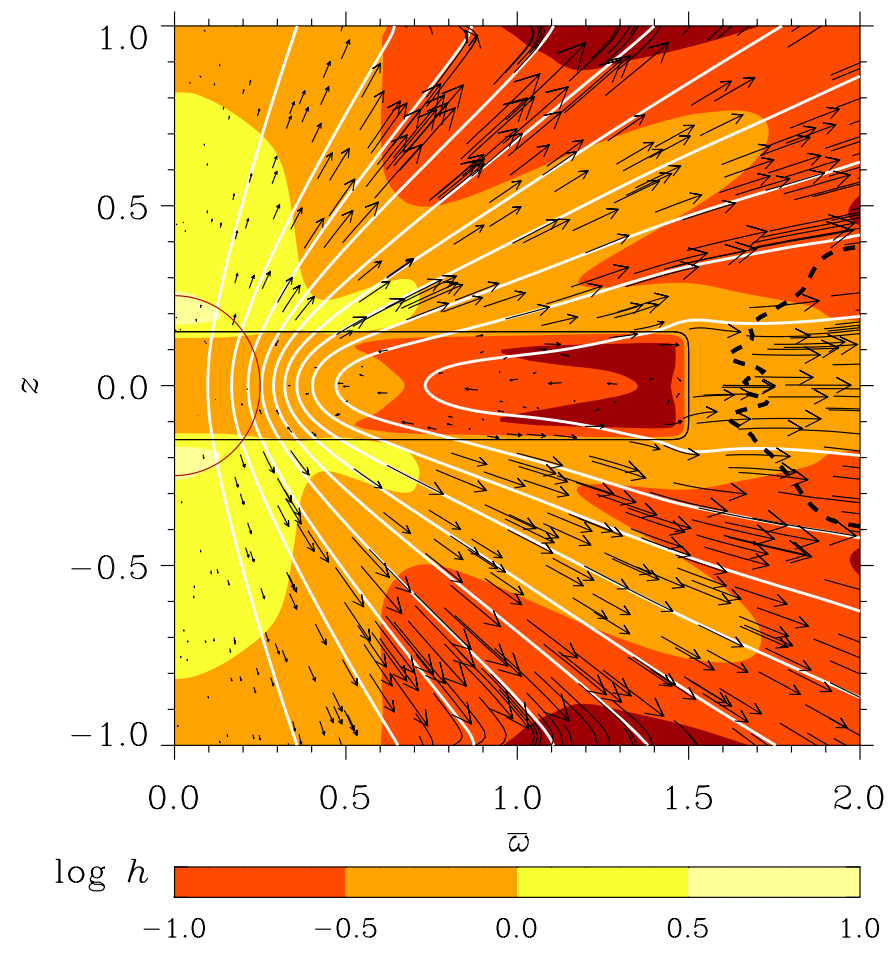

Fig. 21. As in Fig. 7, but with a mass sink at the centre which is five times larger, $r_{0}=0.25$, and $\tau_{\text {star }}=0.112$. Note the absence of outflows along the axis and a larger opening angle of the conical shell, which crosses $z= \pm 1$ at $\varpi \approx 1.2$. Averaged over times $t=500 \ldots 530$, $\beta=0.1$.

was that as much as $30 \%$ of all the matter released in the disc goes into the wind and only about $70 \%$ is accreted by the central object. Earlier estimates (e.g., Pelletier \& Pudritz 1992) indicate that only about $10 \%$ of the matter joins the wind. Another possible deficiency is the fact that in the models presented so far the low-temperature region extends all the way to the stellar surface whilst in reality the cool disc breaks up close to the star because of the stellar magnetic field. Finally, the overall temperature of the disc is generally too high compared with real protostellar discs which are known to have typical temperatures of about a few thousand Kelvin.

The aim of this section is to assess the significance of various improvements in the model related to the above mentioned characteristics. We consider the effect of each of them separately by improving the model step by step.

\subsection{A larger sink}

As discussed in Sects. 3.1 and 3.2, properties of the outflow, especially of the nonmagnetic ones, are sensitive to the parameters of the central sink. It is clear that a very efficient sink would completely inhibit the outflow near the axis. On the other hand, a magnetosphere of the central object can affect the sink efficiency by channelling the flow along the stellar magnetic field (Shu et al. 1994; see also Fendt \& Elstner 1999, 2000). Simulating a magnetosphere turned out to be a difficult task and some preliminary attempts proved unsatisfactory.
Instead, we have considered a model with a geometrically larger sink, and illustrate it in Fig. 21. Here, $r_{0}=0.25$ in Eqs. (5) and (23), which is five times larger than the sink used in our reference model of Fig. 7. The relaxation time of the sink in Eq. (6), $\tau_{\text {star }}$, was rescaled in proportion to the free-fall time at $r_{0}$ as $\tau_{\text {star }} \simeq r_{0} / v_{\mathrm{ff}} \propto r_{0}^{3 / 2}$, which yields $\tau_{\text {star }}=0.112$. The resulting mass loss rate into the wind is $\dot{M}_{\text {wind }} \approx 0.8$, which corresponds to $1.6 \times 10^{-7} M_{\odot} \mathrm{yr}^{-1}$ in dimensional units. Although this is indeed smaller than the value for the reference model $\left(6 \times 10^{-7} M_{\odot} \mathrm{yr}^{-1}\right)$, the overall mass released from the disc is also smaller, resulting in a larger fraction of about $40 \%$ of matter that goes into the wind; only about $60 \%$ is accreted by the central object. As we show below, however, larger accretion fractions can more easily be achieved by making the disc cooler. We conclude that even a sink as large as almost twice the disc half-thickness does not destroy the outflow outside the inner cone. However, the outflow along the axis is nearly completely suppressed.

\subsection{Introducing a gap between the disc and the sink}

In real accretion discs around protostars the disc terminates at some distance away from the star. It would therefore be unrealistic to let the disc extend all the way to the centre. Dynamo action in all our models is restricted to $\varpi \geq 0.2$, and now we introduce an inner disc boundary for the region of lowered entropy as well. In Fig. 22 we present such a model where the $\xi_{\text {disc }}(\boldsymbol{r})$ profile is terminated at $\varpi=0.25$. This inner disc radius affects then not only the region of lowered entropy, but also the distributions of $\alpha, \eta_{\mathrm{t}}, v_{\mathrm{t}}$ and $q_{\varrho}^{\text {disc }}$. The radius of the mass sink was chosen to be $r_{0}=0.15$, i.e. equal to the disc semi-thickness. The correspondingly adjusted value of $\tau_{\text {star }}$ is 0.052. The entropy in the sink is kept as low as that in the disc.

As can be seen from Fig. 22, the gap in the disc translates directly into a corresponding gap in the resulting outflow pattern. At the same time, however, only about $20 \%$ of the released disc material is accreted by the sink and the rest is ejected into the wind. A small fraction of the mass that accretes toward the sink reaches the region near the axis at some distance away from the origin and can then still be ejected as in the models without the gap. Note that our figures (e.g., Fig. 22) show the velocity rather than the azimuthally integrated mass flux density (cf. Fig. 13); the relative magnitude of the latter is much smaller, and so the mass flux from the sink region is not significant.

\subsection{A cooler disc}

We now turn to the discussion of the case with a cooler disc. Numerical constraints prevent us from making the entropy gradient between disc and corona too steep. Nevertheless, we were able to reduce the value of $\beta$ down to 0.005 , which is 20 times smaller than the value used for the reference model in Fig. 7. The $\beta$ value for the sink was reduced to 0.02 ; smaller values proved numerically difficult. 


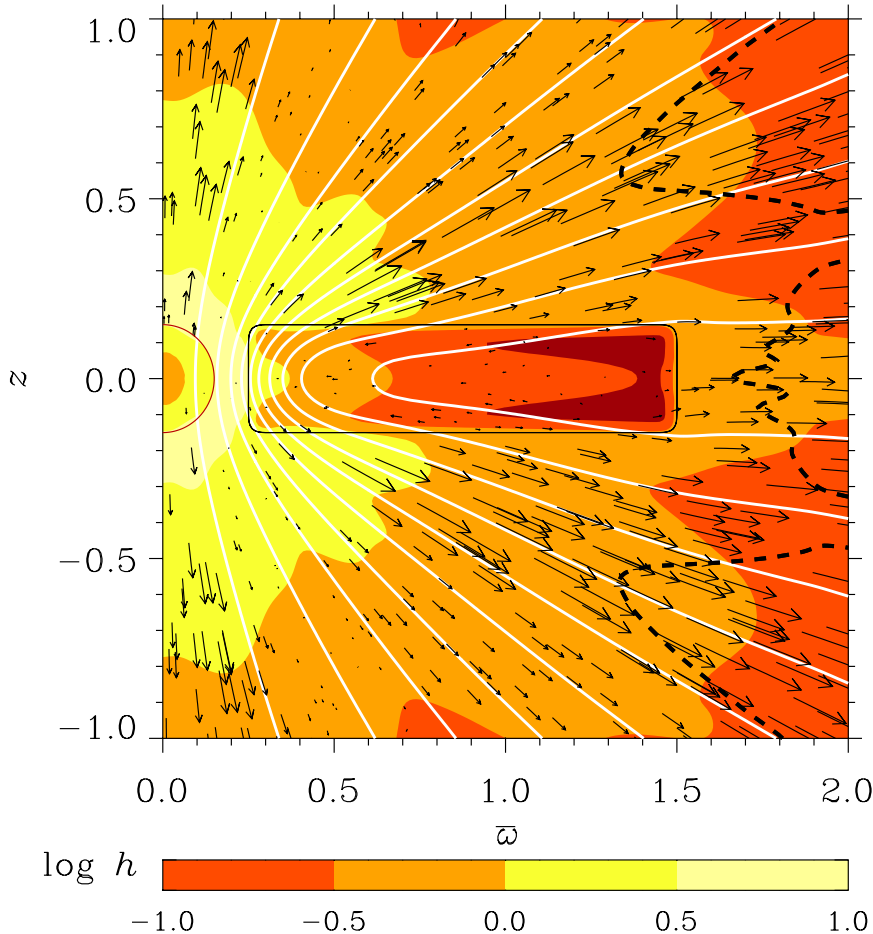

Fig. 22. As in Fig. 7, but with a gap between the sink and the disc with the disc inner radius at $\varpi=0.25$. The radius of the stellar mass sink is three times larger, $r_{0}=0.15$, and $\tau_{\text {star }}=0.052$. Outflow is absent along field lines passing through the gap. Averaged over times $t=400 \ldots 660, \beta=0.1$ in both the disc and the sink.

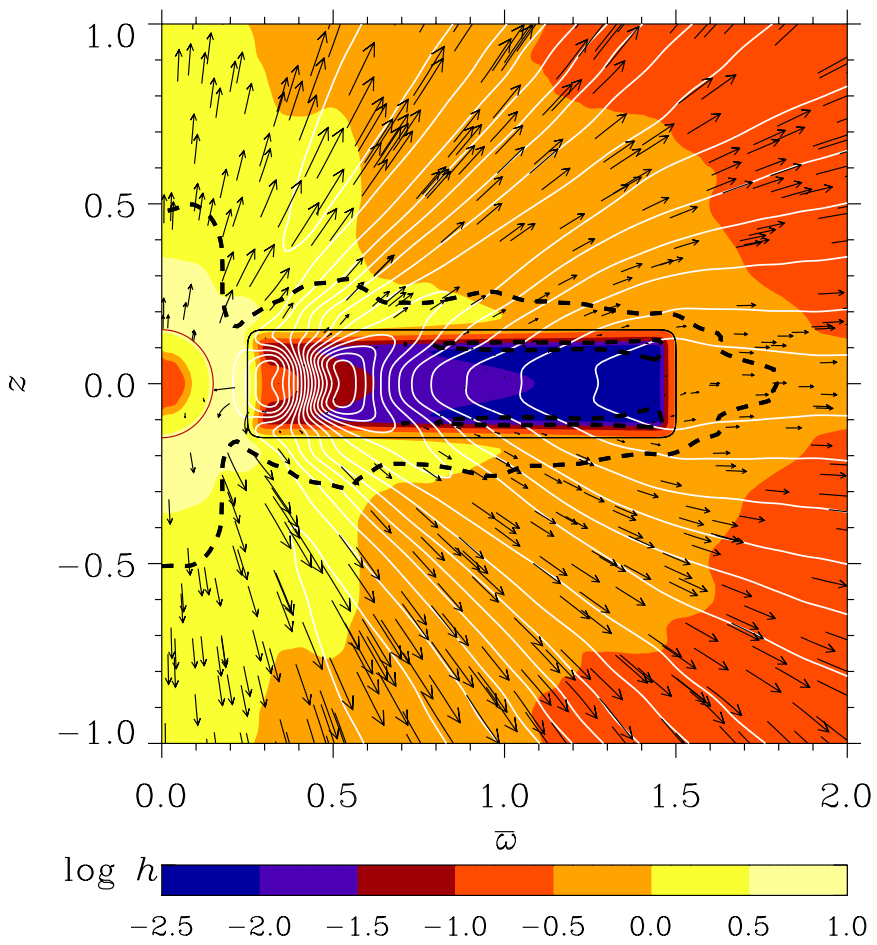

Fig. 23. As in Fig. 22, but with $\beta=0.005$ in the disc and $\beta=0.02$ in the sink. Averaged over times $t=150 \ldots 280$ when the overall magnetic activity in the disc is relatively low.

The value of $\beta=0.005$ results in a disc temperature of $3 \times 10^{3} \mathrm{~K}$ in the outer parts and $1.5 \times 10^{4} \mathrm{~K}$ in the inner

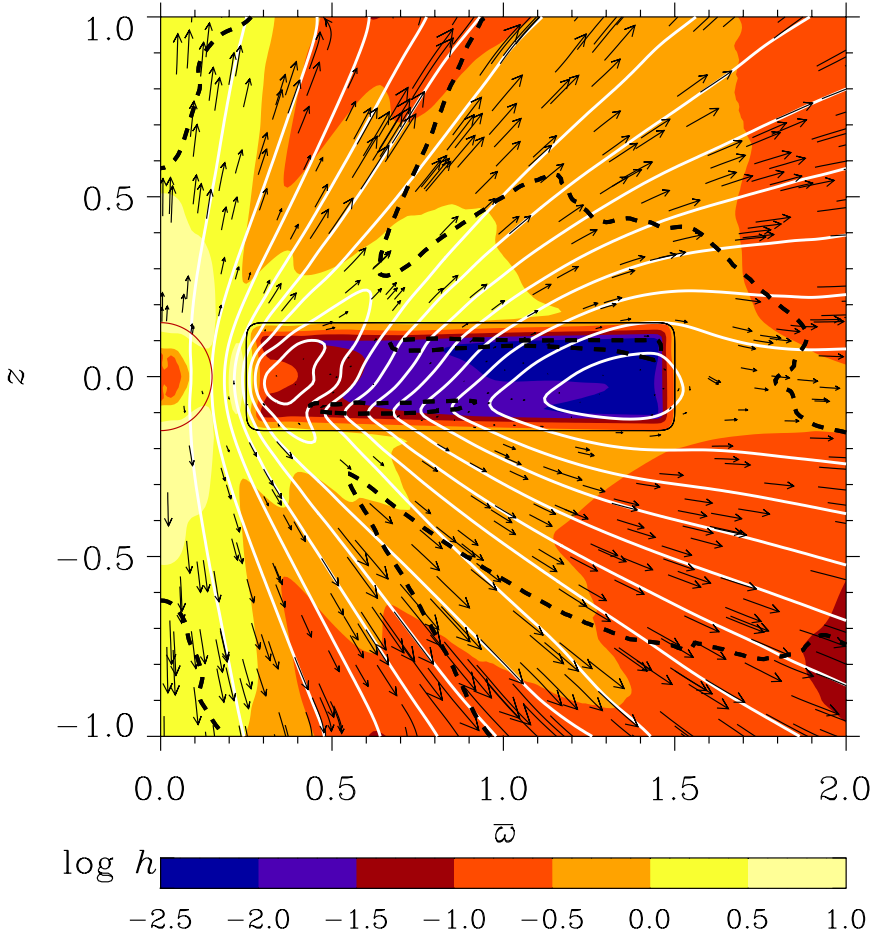

Fig. 24. As in Fig. 23, but averaged over a period of enhanced magnetic activity in the disc, $t=300 \ldots 320$. A conical shell develops which crosses $z= \pm 1$ at $\varpi \approx 0.6$.

parts. As in Sect. 4.2, the disc terminates at an inner radius of $\varpi=0.25$. At $\varpi \approx 0.5$, the density in dimensional units is about $10^{-9} \mathrm{~g} \mathrm{~cm}^{-3}$, which is also the order of magnitude found for protostellar discs. The resulting magnetic field and outflow geometries are shown in Figs. 23 and 24.

A characteristic feature of models with a cooler disc is a more vigorous temporal behaviour with prolonged episodes of reduced overall magnetic activity in the disc during which the Alfvénic surface is closer to the disc surface, and phases of enhanced magnetic activity where the Alfvénic surface has moved further away. Figures 23 and 24 are representative of these two states. It is notable that the structured outflow of the type seen in the reference model occurs in states with strong magnetic field and disappears during periods with weak magnetic field.

Another interesting property of the cooler discs is that now a smaller fraction of matter goes into the wind (10-20\%), and $80-90 \%$ is accreted by the central object, in a better agreement with the estimates of Pelletier \& Pudritz (1992).

We note in passing that channel flow solutions typical of two-dimensional simulations of the magneto-rotational instability (Hawley \& Balbus 1991) are generally absent in the simulations presented here. This is because the magnetic field saturates at a level close to equipartition between magnetic and thermal energies. The vertical wavelength of the instability can then exceed the half-thickness of the disc. In some of our simulations, indications of channel flow behaviour still can be seen. An example is Fig. 23 where the magnetic energy is weak 


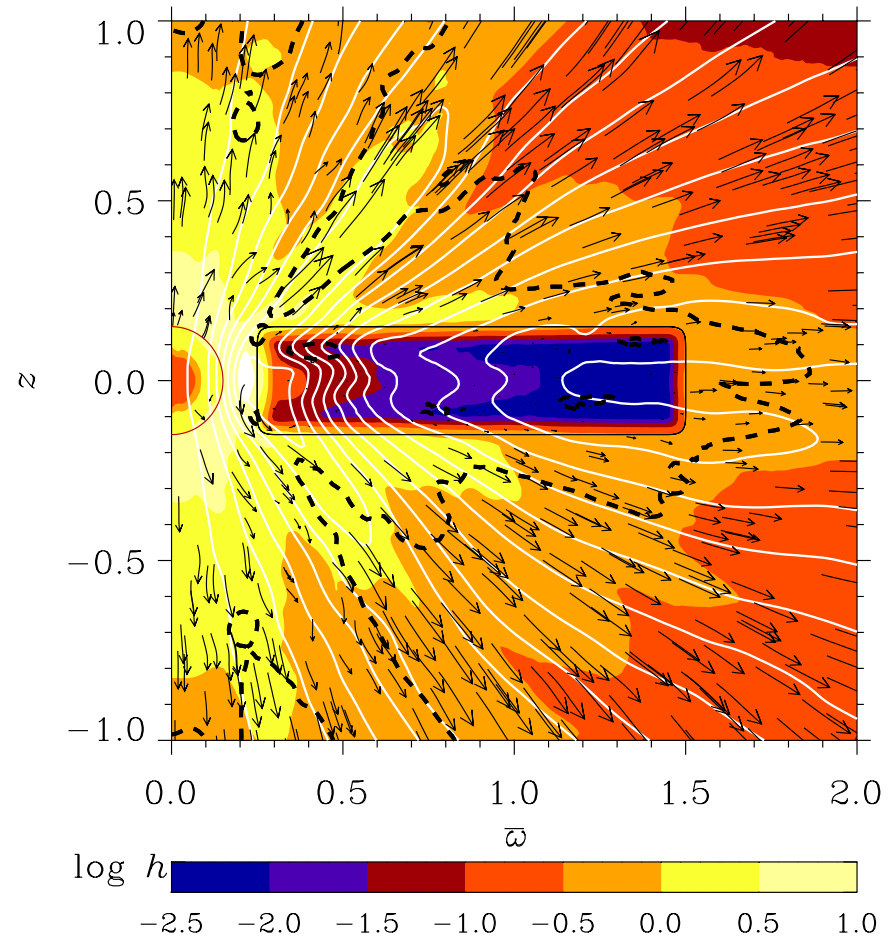

Fig. 25. As in Figs. 23 and 24, but with $\eta_{0}=2 \times 10^{-5}, \eta_{\mathrm{t} 0}=4 \times 10^{-5}$, $c_{v}^{\text {adv }}=0.002$, and $\alpha_{0}=-0.15$. Averaged over times $t=230 \ldots 236$ (where the magnetic energy is enhanced), $\alpha_{\mathrm{SS}}=0.004$.

enough so that the magneto-rotational instability is not yet suppressed.

According to the Shakura-Sunyaev prescription, turbulent viscosity and magnetic diffusivity are reduced in a cooler disc because of the smaller sound speed (cf. Eq. (10)). Since in the corona the dominant contributions to the artificial advection viscosity $v_{\text {adv }}$ come from the poloidal velocity and poloidal Alfvén speed (and not from the sound speed), $c_{v}^{\text {adv }}$ has to be reduced. Here we choose $\alpha_{\mathrm{SS}}=0.004$ and reduce $c_{v}^{\text {adv }}$ by a factor of 10 to $c_{v}^{\text {adv }}=0.002$. Since we do not explicitly parameterize the turbulent magnetic diffusivity $\eta_{\mathrm{t}}$ with the sound speed (cf. Eq. (15)), also $\eta_{t 0}$ needs to be decreased, together with the background diffusivity $\eta_{0}$. We choose here values that are 25 times smaller compared to the previous runs, i.e. $\eta_{\mathrm{t} 0}=4 \times 10^{-5}$ and $\eta_{0}=2 \times 10^{-5}$, which corresponds to $\alpha_{\mathrm{SS}}^{(\eta)}$ ranging between 0.001 and 0.007 . With this choice of coefficients, the total viscosity and magnetic diffusivity in the disc have comparable values of a few times $10^{-5}$.

The effect of reduced viscosity and magnetic diffusivity is shown in Fig. 25. Features characteristic of the channel flow solution are now present, because the vertical wavelength of the magneto-rotational instability is here less than the halfthickness of the disc.

\section{Discussion}

If the disc dynamo is sufficiently strong, our model develops a clearly structured outflow which is fast, cool and rarefied within a conical shell near the rotation axis where most of the angular momentum and magnetic energy is carried, and is slower, hotter and denser in the region around the axis as well as in the outer parts of the domain. The slower outflow is driven mostly by the entropy contrast between the disc and the corona, but the faster wind within the conical shell is mostly driven magnetocentrifugally. Without a central mass sink, the flow near the axis is faster, but otherwise the flow structure is similar to that with the sink.

The half-opening angle of the cone with hot, dense gas around the axis is about $20^{\circ}-30^{\circ}$; this quantity somewhat changes with model parameters but remains close to that range. The outflow in our models does not show any signs of collimation. It should be noted, however, that not all outflows from protostellar discs are actually collimated, especially not at such small distance from the source. An example is the Becklin-Neugebauer/Kleinmann-Low (BN/KL) region in the Orion Nebula (Greenhill et al. 1998), which has a conical outflow with a half-opening angle of $30^{\circ}$ out to a distance of 25 $60 \mathrm{AU}$ from its origin. Therefore, collimation within a few $\mathrm{AU}$ (the size of our computational domain) is expected to be only weak.

The region around the fast, cool and rarefied conical shell seen in Fig. 7 is similar to the flow structure reported by Krasnopolsky et al. (1999); see their Fig. 1. In their model, however, the thin axial jet was caused by an explicit injection of matter from the inner parts of the disc which was treated as a boundary. In our reference model the fast outflow is subAlfvénic because of the presence of a relatively strong poloidal field, whereas in Krasnopolsky et al. (1999) the outflow becomes super-Alfvénic at smaller heights. Outside the conical shell the outflow is mainly pressure driven, even though the criterion of Blandford \& Payne (1982) is fulfilled. However, as Casse \& Ferreira (2000b) pointed out, pressure driven outflows might dominate over centrifugally driven outflows if thermal effects are strong enough.

In our model, matter is replenished in the resolved disc in a self-regulatory manner where and when needed. We believe that this is an improvement in comparison to the models of Ouyed \& Pudritz (1997a, 1997b, 1999) and Ustyugova et al. (1995), where mass inflow is prescribed as a boundary condition at the base of the corona. If we put $q_{Q}^{\text {disc }}=0$ in Eqs. (1) and (7), the disc mass soon drops to low values and the outflow ceases. This is qualitatively the same behaviour as in the models of, e.g., Kudoh et al. (1998).

We should stress the importance of finite magnetic diffusivity in the disc: although poloidal velocity and poloidal magnetic field are well aligned in most of the corona, dynamo action in the disc is only possible in the presence of finite magnetic diffusivity, and the flow can enter the corona only by crossing magnetic field lines in the disc.

An outflow occurs in the presence of both dipolar and quadrupolar type magnetic fields, even though fields with dipolar symmetry seem to be more efficient in magneto-centrifugal driving (cf. von Rekowski et al. 2000). The effects of the magnetic parity on the outflow structure deserves further analysis.

The dynamo active accretion disc drives a significant outward Poynting flux in our model. Assuming that this applies 
equally to protostellar and AGN discs, this result could be important for understanding the origin of seed magnetic fields in galaxies and galaxy clusters; see Jafelice \& Opher (1992) and Brandenburg (2000) for a discussion. We note, however, that the pressure of the intracluster gas may prevent the magnetized plasma from active galactic nuclei to spread over a significant volume (Goldshmidt \& Rephaeli 1994).

Our model can be improved in several respects. In many systems, both dynamo-generated and external magnetic fields may be present, so a more comprehensive model should include both. We used an $\alpha^{2} \Omega$ dynamo to parameterize magnetic field generation in the disc because we have restricted ourselves to axisymmetric models. As argued by Brandenburg (1998), dynamo action of turbulence which is driven by the magneto-rotational instability can be roughly described as an $\alpha^{2} \Omega$ dynamo. But this parameterization can be relaxed in threedimensional simulations where one may expect that turbulence will be generated to drive dynamo action. Such simulations will be discussed elsewhere (von Rekowski et al. 2002).

Since our model includes angular momentum transport by both viscous and magnetic stresses, it is natural that the accreted matter is eventually diverted into an outflow near the axis; this is further facilitated by our prescribed entropy gradient at the disc surface. We believe that this picture is physically well motivated (Bell \& Lucek 1995), with the only reservation that we do not incorporate the (more complicated) physics of coronal heating and disc cooling, but rather parameterize it with a fixed entropy contrast. We include a mass sink at the centre which could have prevented the outflow, and indeed the sink strongly affects nonmagnetized outflows. We have shown, however, that the magnetic field can efficiently shield the sink and thereby support a vigorous disc wind.

The assumption of a prescribed entropy distribution is a useful tool to control the size of the disc and to parameterize the heating of the disc corona. However, it should be relaxed as soon as the disc physics can be described more fully. The energy equation, possibly with radiation transfer, should be included. This would lead to a self-consistent entropy distribution and would admit the deposition of viscous and Ohmic heat in the outflow. In the simulations by von Rekowski et al. (2002), entropy is evolved.

We believe that a mass source is a necessary feature of any model of this kind if one wishes to obtain a steady state. In the present paper the mass source is distributed throughout the whole disc to represent replenishment of matter from the midplane of the disc. Alternatively, a mass source could be located near to or on the domain boundary.

Acknowledgements. We are grateful to C. G. Campbell, R. Henriksen, J. Heyvaerts, R. Ouyed and R. E. Pudritz for fruitful discussions. We acknowledge many useful comments of the anonymous referee. This work was partially supported by PPARC (Grants PPA/G/S/1997/00284 and 2000/00528) and the Leverhulme Trust (Grant F/125/AL). Use of the PPARC supported supercomputers in St Andrews and Leicester is acknowledged.

\section{References}

Bardou, A., von Rekowski, B., Dobler, W., et al. 2001, A\&A, 370, 635 Beck, R., Brandenburg, A., Moss, D., et al. 1996, ARA\&A, 34, 155 Bell, A. R. 1994, Phys. Plasmas, 1, 1643

Bell, A. R., \& Lucek, S. G. 1995, MNRAS, 277, 1327

Blandford, R. D., \& Payne, D. R. 1982, MNRAS, 199, 883

Brandenburg, A. 1998, in Theory of Black Hole Accretion Discs, ed. M. A. Abramowicz, G. Björnsson, \& J. E. Pringle (Cambridge Univ. Press)

Brandenburg, A. 2000, Phil. Trans. Roy. Soc. Lond. A, 358, 759

Brandenburg, A. 2003, in Advances in Non-linear Dynamos, ed. A. Ferriz-Mas, \& M. Núñez Jiménez (Taylor \& Francis), in press [astro-ph/0109497]

Brandenburg, A., Nordlund, Å, Stein, R. F., et al. 1995, ApJ, 446, 741

Brandenburg, A., Tuominen, I., \& Krause, F. 1990, GAFD, 50, 95

Campbell, C. G. 1999, MNRAS, 310, 1175

Campbell, C. G. 2000, MNRAS, 317, 501

Campbell, C. G. 2001, MNRAS, 323, 211

Casse, F., \& Ferreira, J. 2000a, A\&A, 353, 1115

Casse, F., \& Ferreira, J. 2000b, A\&A, 361, 1178

Feigelson, E. D., \& Montmerle, T. 1999, ARA\&A, 37, 363

Fendt, C., \& Elstner, D. 1999, A\&A, 349, L61

Fendt, C., \& Elstner, D. 2000, A\&A, 363, 208

Ferreira, J. 1997, A\&A, 319, 340

Ferreira, J., \& Pelletier, G. 1995, A\&A, 295, 807

Fromang, S., Terquem, C., \& Balbus, S. A. 2002, MNRAS, 329, 18

Fukue, J. 1989, PASJ, 41, 123

Galeev, A. A., Rosner, R., \& Vaiana, G. S. 1979, ApJ, 229, 318

Glassgold, A. E., Feigelson, E. D., \& Montmerle, T. 2000, in Protostars and Planets IV, ed. V. Mannings, A. Boss, \& S. Russell (Univ. Arizona Press)

Goldshmidt, O., \& Rephaeli, Y. 1994, ApJ, 431, 586

Goodson, A. P., Winglee, R. M., \& Böhm, K.-H. 1997, ApJ, 489, 199

Greenhill, L. J., Gwinn, C. R., Schwartz, C., et al. 1998, Nature, 396, 650

Grosso, N., Montmerle, T., Bontemps, S., et al. 2000, A\&A, 359, 113

Hawley, J. F., \& Balbus, S. A. 1991, ApJ, 376, 223

Hawley, J. F., Gammie, C. F., \& Balbus, S. A. 1996, ApJ, 464, 690

Heyvaerts, J., Priest, E. R., \& Bardou, A. 1996, ApJ, 473, 403

Jafelice, L. C., \& Opher, R. 1992, MNRAS, 257, 135

Königl, A., \& Pudritz, R. E. 2000, in Protostars and Planets IV, ed. V. Mannings, A. Boss, \& S. Russell (Univ. Arizona Press)

Krasnopolsky, R., Li, Z.-Y., \& Blandford, R. 1999, ApJ, 526, 631

Krause, F., \& Rädler, K.-H. 1980, Mean-Field Magnetohydrodynamics and Dynamo Theory (Akademie-Verlag, Berlin)

Kudoh, T., Matsumoto, R., \& Shibata, K. 1998, ApJ, 508, 186

Kwan, J. 1997, ApJ, 489, 284

Kwan, J., \& Tademaru, E. 1995, ApJ, 454, 382

Lubow, S. H., Papaloizou, J. C. B., \& Pringle, J. E. 1994, MNRAS, 267,235

Lucek, S. G., \& Bell, A. R. 1996, MNRAS, 281, 245

Lucek, S. G., \& Bell, A. R. 1997, MNRAS, 290, 327

Matsumoto, R., Uchida, Y., Hirose, S., et al. 1996, ApJ, 461, 115

Mestel, L. 1999, Stellar Magnetism (Clarendon Press, Oxford)

Moss, D., Shukurov, A., \& Sokoloff, D. 1999, A\&A, 343, 120

Ouyed, R., Clarke, D. A., \& Pudritz, R. E. 2003, ApJ, 582, 292

Ouyed, R., \& Pudritz, R. E. 1997a, ApJ, 482, 712

Ouyed, R., \& Pudritz, R. E. 1997b, ApJ, 484, 794

Ouyed, R., \& Pudritz, R. E. 1999, MNRAS, 309, 233

Ouyed, R., Pudritz, R. E., \& Stone, J. M. 1997, Nature, 385, 409

Papaloizou, J. C. B., \& Terquem, C. 1999, ApJ, 521, 823

Parker, E. N. 1994, Spontaneous current sheets in magnetic fields (Oxford University Press, Oxford) 
Pelletier, G., \& Pudritz, R. E. 1992, ApJ, 394, 117

Pudritz, R. E. 1981a, MNRAS, 195, 881

Pudritz, R. E. 1981b, MNRAS, 195, 897

von Rekowski, B., Brandenburg, A., Dobler, W., et al. 2002, in preparation

von Rekowski, M., Rüdiger, G., \& Elstner, D. 2000, A\&A, 353, 813

Romanova, M. M., Ustyugova, G. V., Koldoba, A. V., et al. 1997, ApJ, 482, 708

Romanova, M. M., Ustyugova, G. V., Koldoba, A. V., et al. 1998, ApJ, 500,703

Ruzmaikin, A. A., Shukurov, A. M., \& Sokoloff, D. D. 1988, Magnetic Fields of Galaxies (Kluwer Academic Publishers, Dordrecht)

Shalybkov, D., \& Rüdiger, G. 2000, MNRAS, 315, 762
Shu, F., Najita, J., Ostriker, E., et al. 1994, ApJ, 429, 781

Stepinski, T., \& Levy, E. H. 1988, ApJ, 331, 416

Stone, J. M., \& Norman, M. L. 1994, ApJ, 433, 746

Takahara, F., Rosner, R., \& Kusunose, M. 1989, ApJ, 346, 122

Thomsen, F., \& Nordlund, Å. 2003, A\&A, submitted [astro-ph/0206394]

Uchida, Y., \& Shibata, K. 1985, PASJ, 37, 515

Urpin, V., \& Brandenburg, A. 1998, MNRAS, 294, 399

Ustyugova, G. V., Koldoba, A. V., Romanova, M. M., et al. 1995, ApJ, 439, L39

Wardle, M., \& Königl, A. 1993, ApJ, 410, 218

Ziegler, U., \& Rüdiger, G. 2000, A\&A, 356, 1141 